\title{
Peer-to-peer meets wireless: Opportunities and challenges
}

\author{
Antony Rowstron
}

Microsoft Research, Cambridge 


\section{Talk outline}

- Introduction (Overlays, underlays and wireless )

- Wireless routing inspired by structured overlays

- Mesh networks

- Design of Virtual Ring Routing (VRR)

- Evaluation of VRR

- Vehicular Networking

- Design concepts of PVRP

- Preliminary results 


\section{Structured overlays/DHTs}

- Experiences learnt with structured overlays

- Self-organizing

- Fault-tolerant

- Scalable

- Decentralized

- Performance

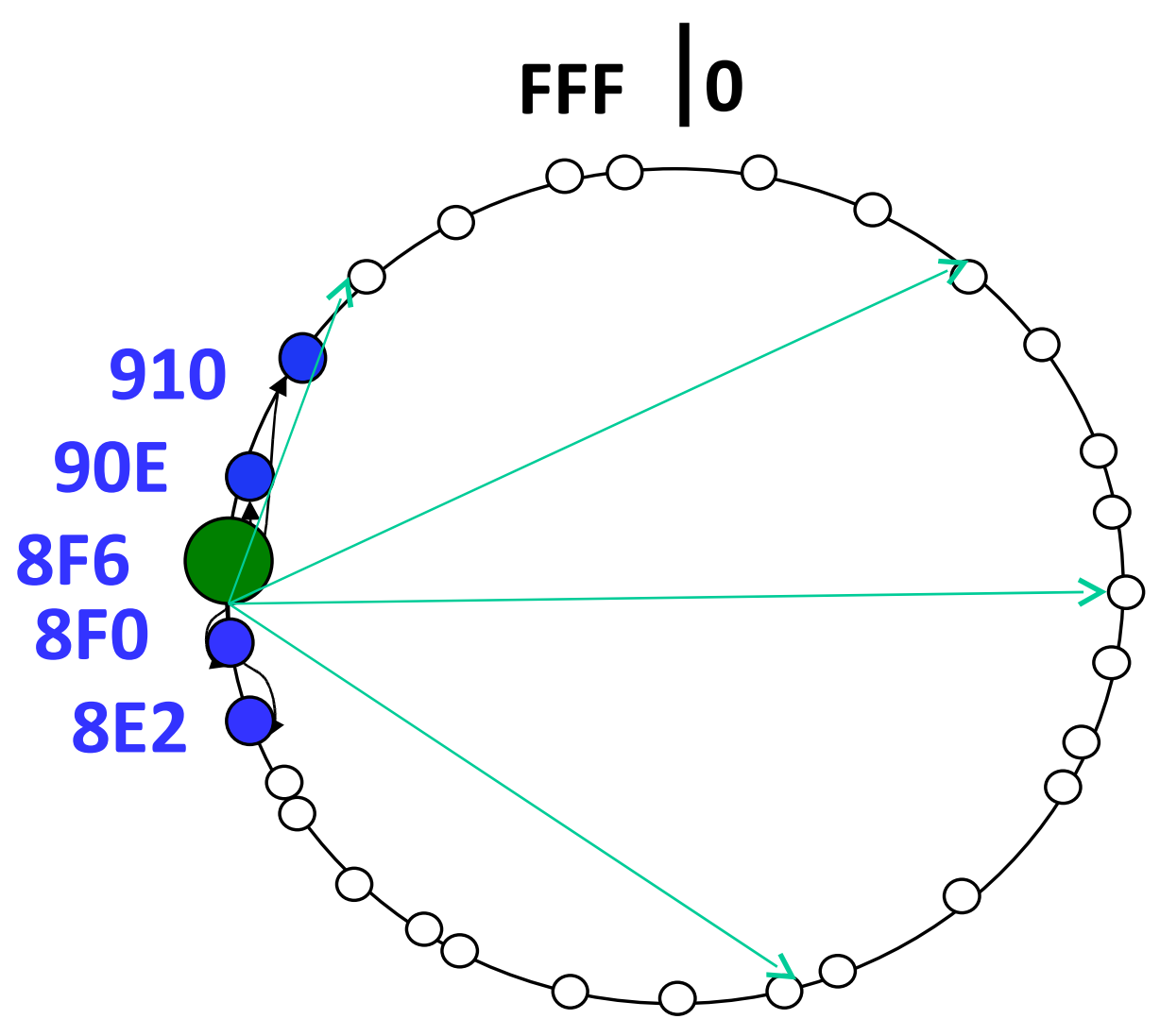




\section{Structured overlays/DHTs}

- Experiences learnt with structured overlays

- Self-organizing

- Fault-tolerant

- Scalable

- Decentralized

- Performance

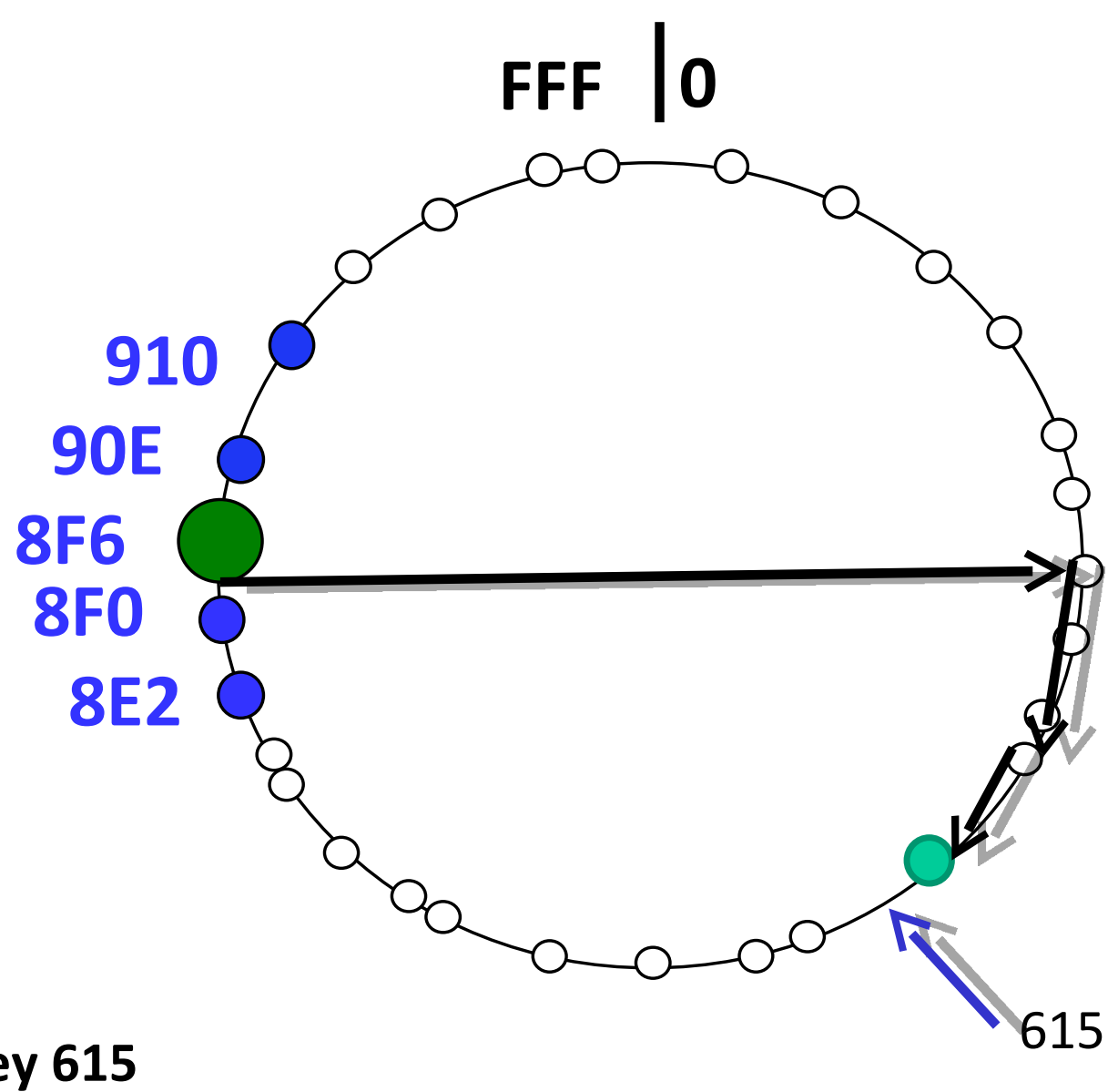

route to key 615 


\section{Wireless routing}

- Mesh networks beginning to be deployed:

- Office buildings

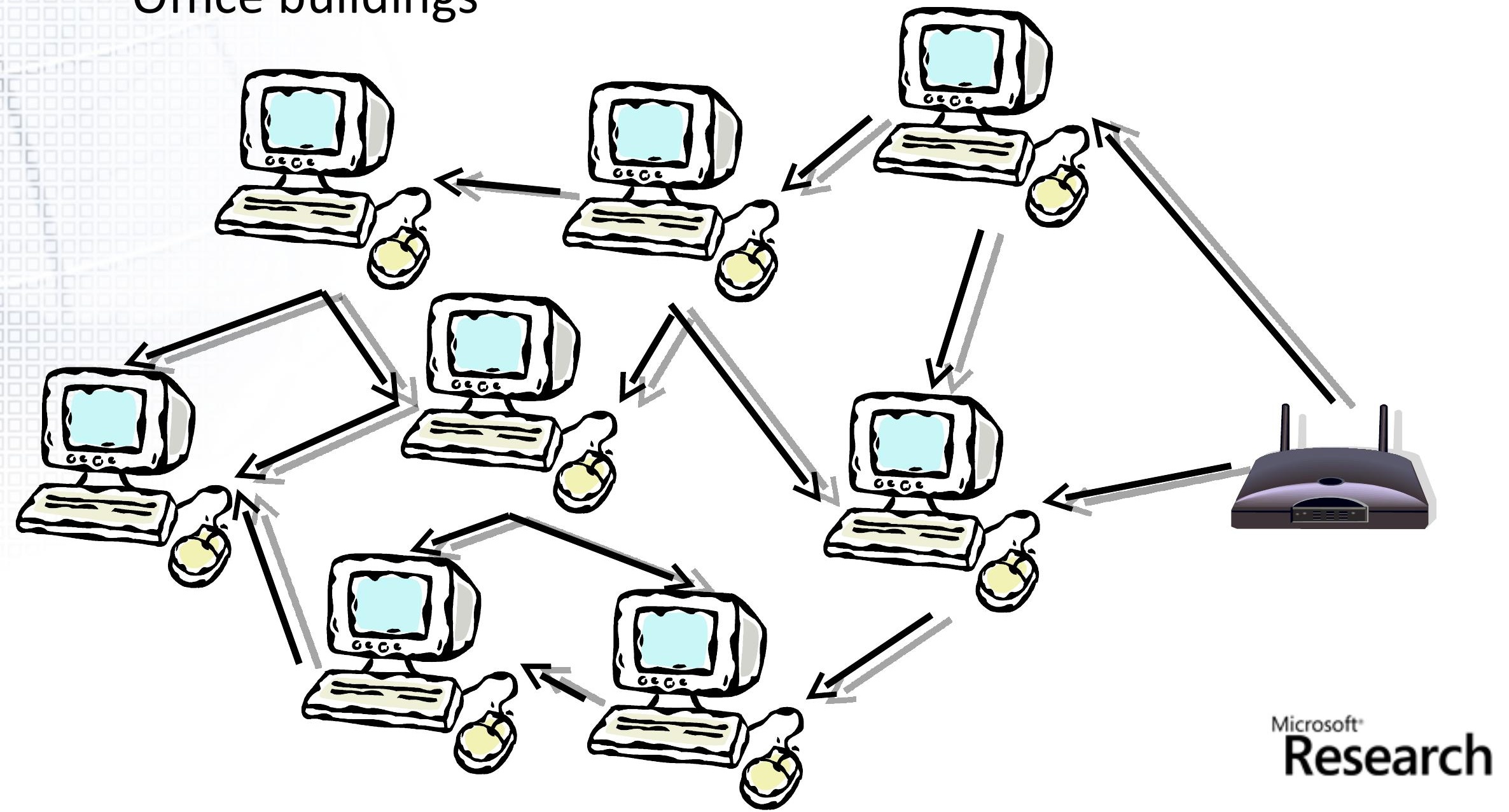




\section{Proactive routing}

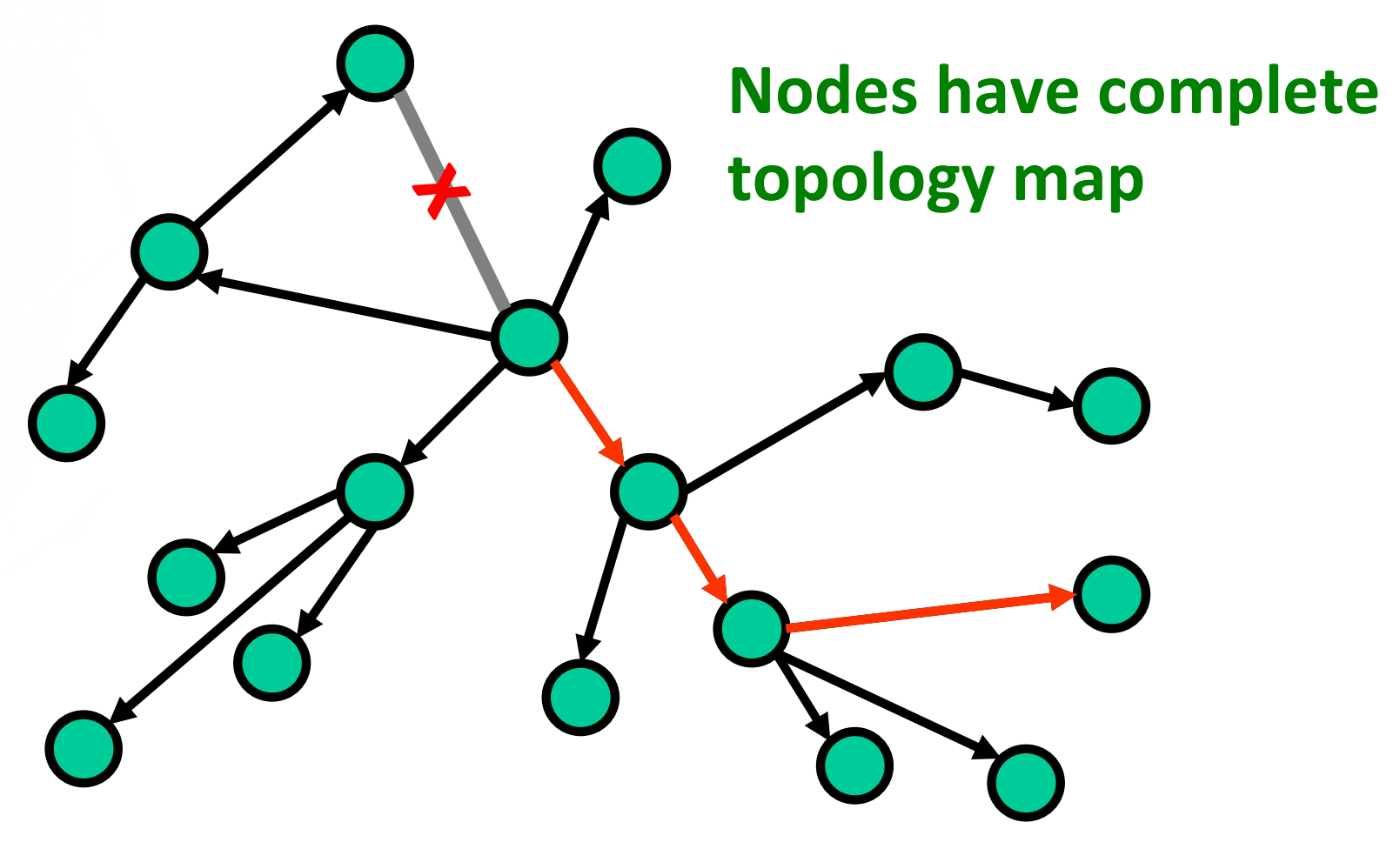

For example: OLSR and DSDV 


\section{Reactive routing}

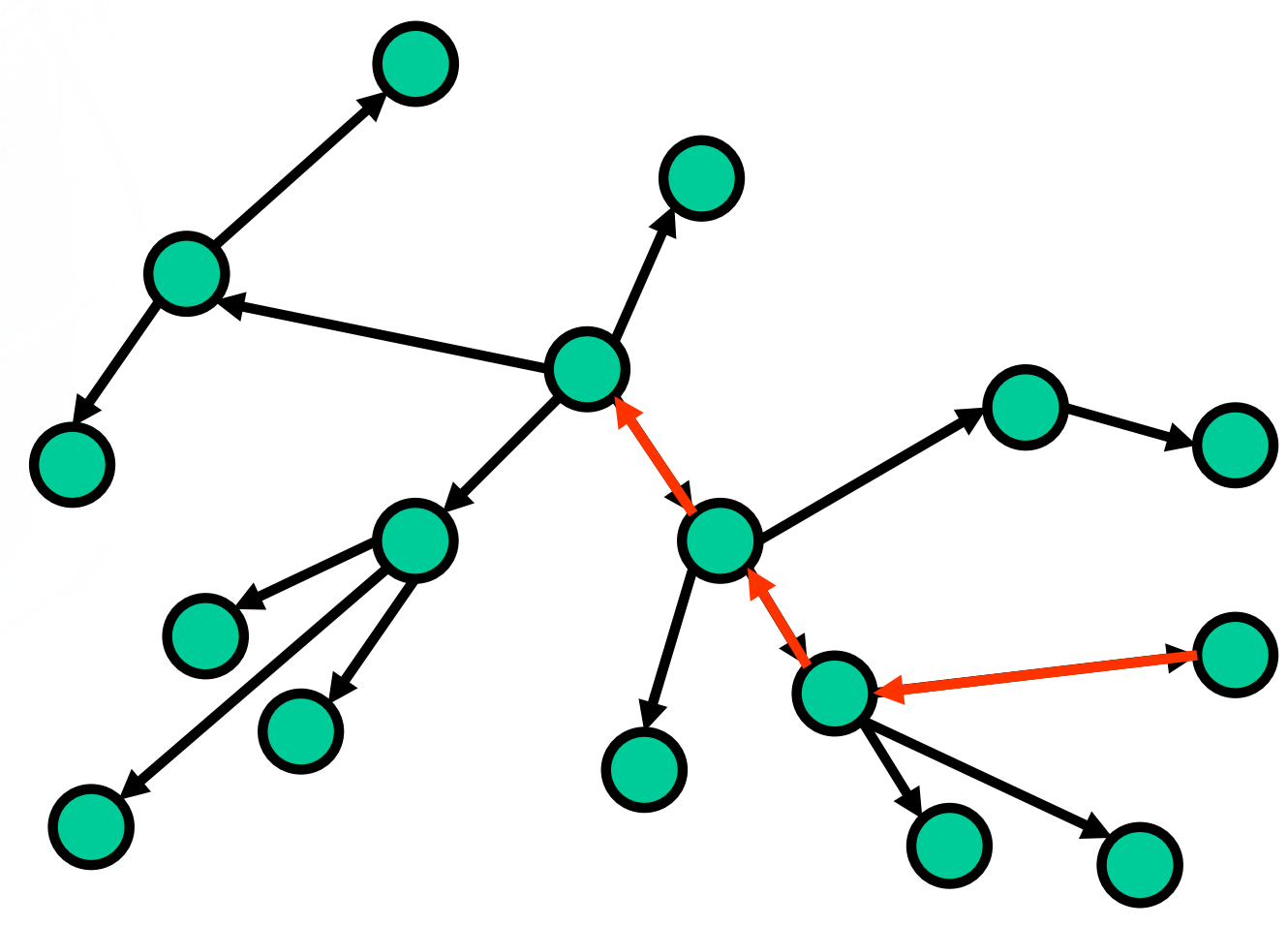

For example: DSR and AODV 


\section{Coordinate-based routing}

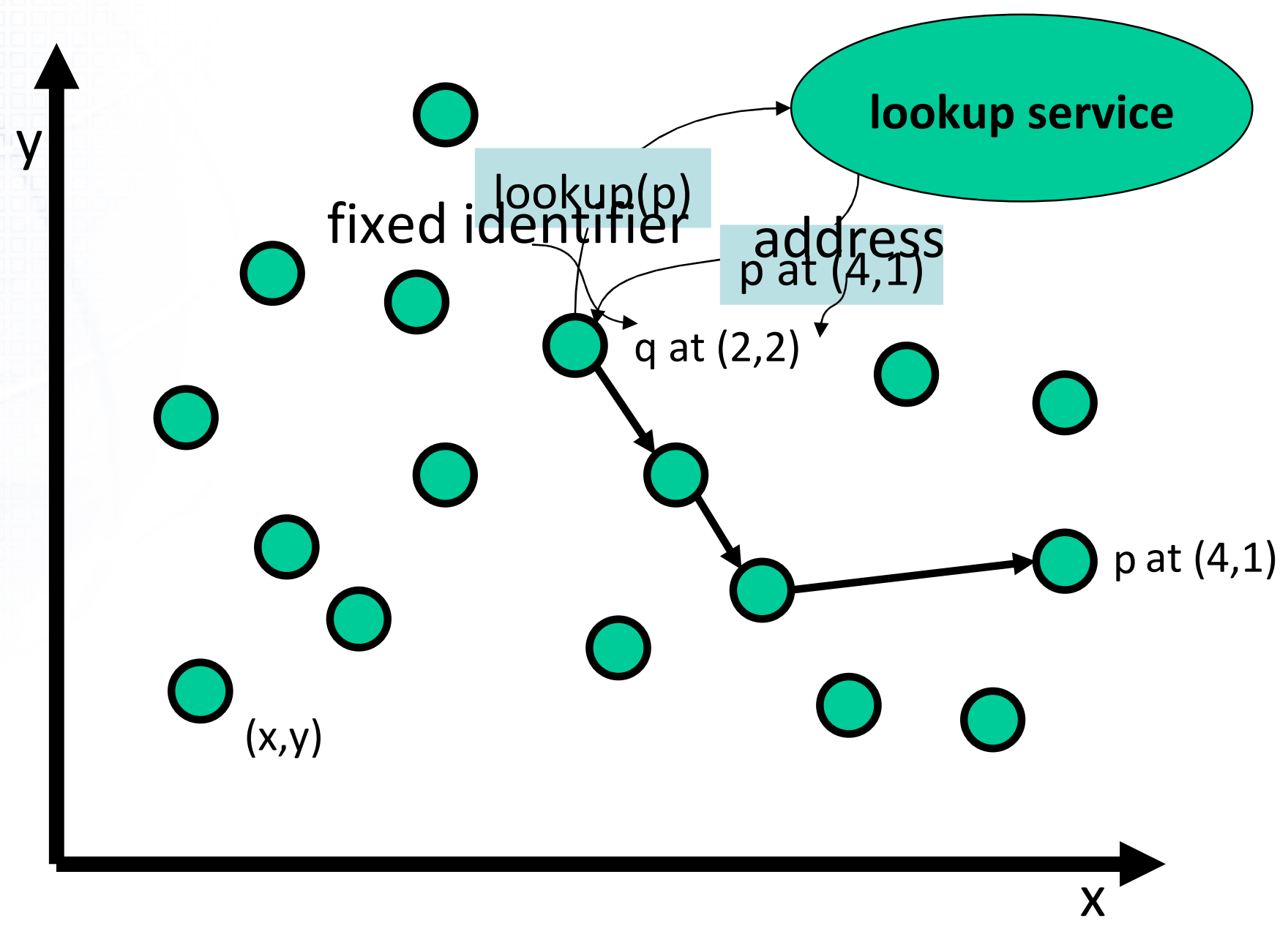

For example: GPSR and BVR 


\section{Prior work on wireless routing}

- Flooding based algorithms scale poorly

- Proactive algorithms flood on topology changes

- On demand algorithms flood to discover routes

- Geographical and landmark routing

- Scale well but nodes have identifier and address

- Some apps (sensor networks) may require just address

- DHT-like structure to translate between the two

- Route setup delays

- Additional maintenance overhead

- Another target for attacks 


\section{Virtual Ring Routing}

Joint work with Miguel Castro, Greg O'Shea plus interns

- Virtual Ring Routing - protocol inspired by DHT design

- Single fixed identifier

- No flooding

- Provides DHT for free

- Strict layering on wireless routing is inefficient

- Poor interaction between DHT and routing layer 


\section{VRR: The virtual ring}

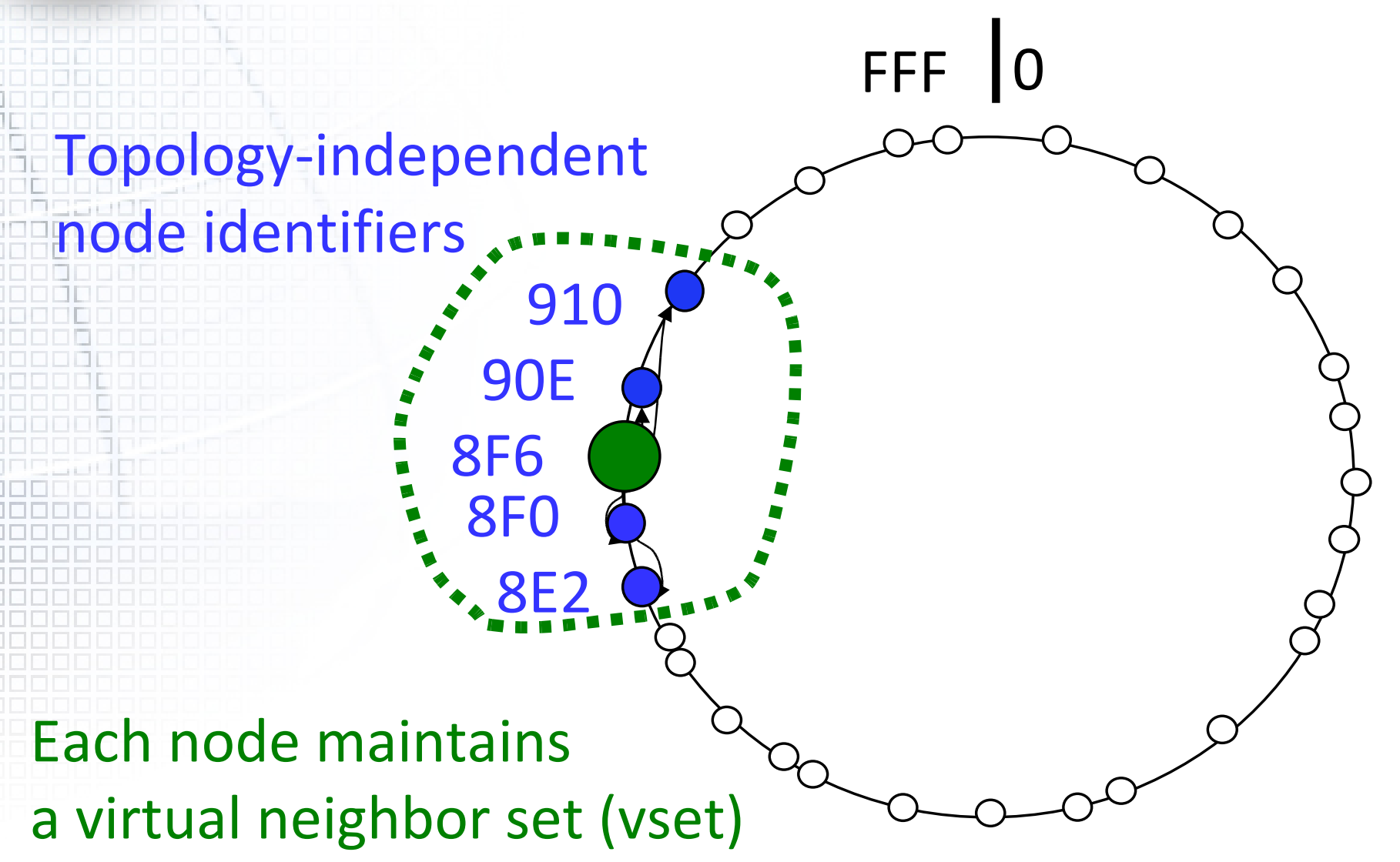

Nodes organized into virtual ring by increasing identifier value 


\section{VRR: Routing paths}

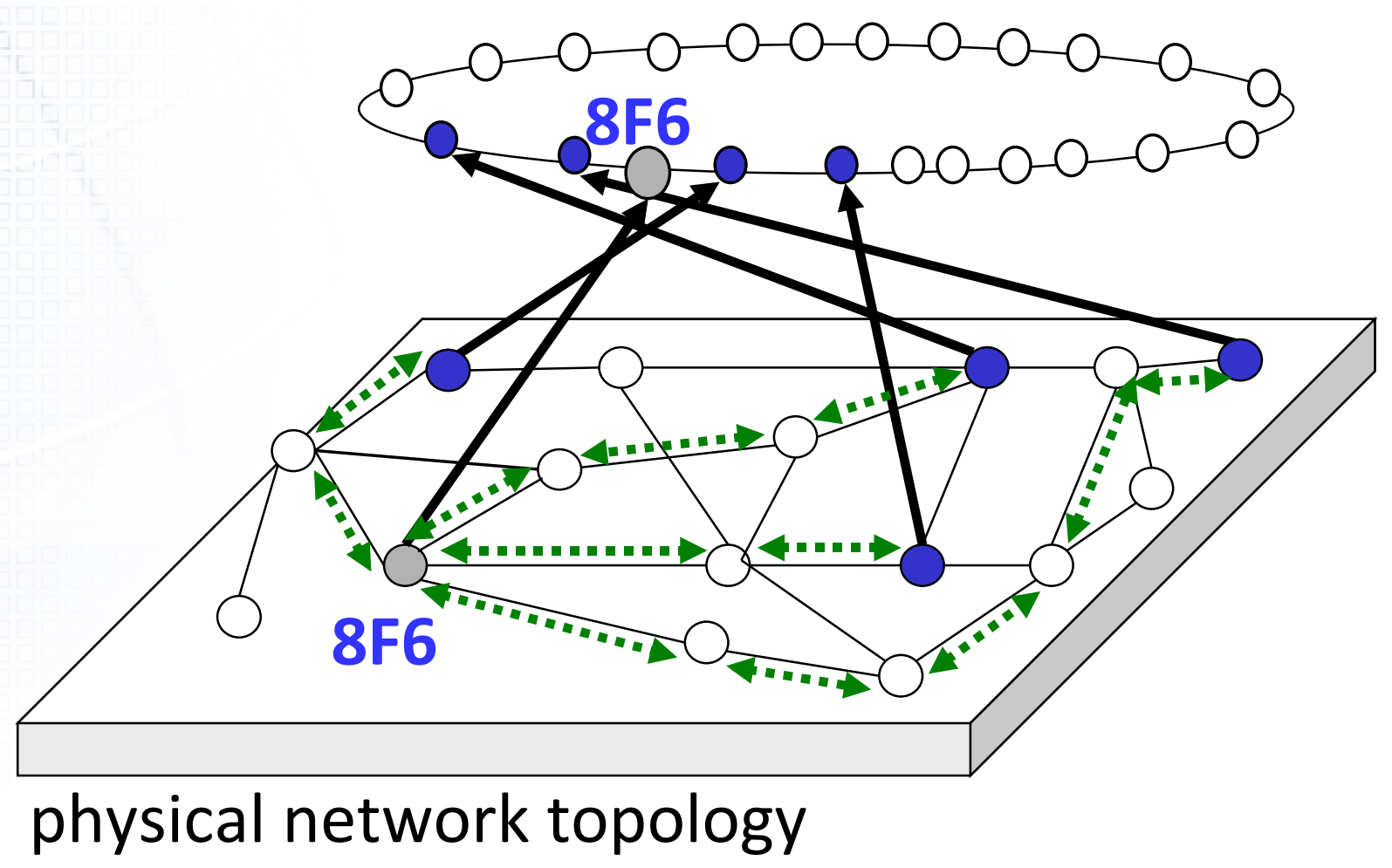

Nodes only maintain routing paths to virtual neighbors:

- Paths maintained proactively

- Paths are bidirectional and typically multi-hop 


\section{VRR: Forwarding table}

\begin{tabular}{|c|c|c|c|c|}
\hline endpointA & endpointB & nextA & nextB & pathld \\
\hline $8 F 6$ & $90 E$ & me & F42 & 31 \\
\hline 910 & $8 F 6$ & $10 E$ & me & 10 \\
\hline $14 A$ & 140 & $F 42$ & $10 E$ & 2 \\
\hline $8 F 6$ & F42 & me & F42 & FF \\
\hline
\end{tabular}

forwarding table for node 8F6

- Paths recorded in forwarding tables along path

- Forwarding table contains

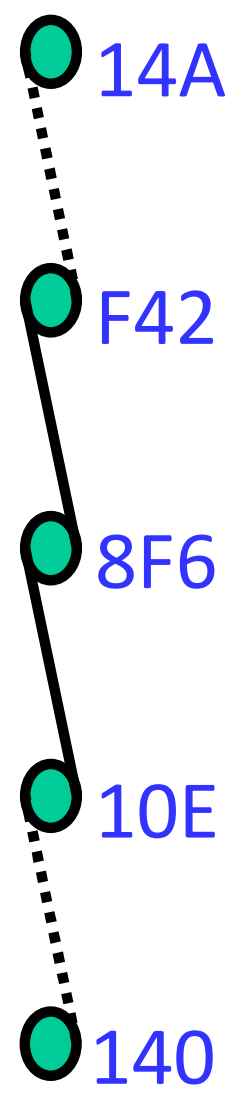

- Paths between node and vset members

- Paths between other nodes that go through node

- Paths to physical neighbors 


\section{VRR: Routing}

- Forward message destined to $x$ by

- Picking endpoint $e$ numerically closest to $x$

- Forwarding message to next hop towards $e$

- Deliver message to node with id closest to $x$ 


\section{VRR: Example routing}

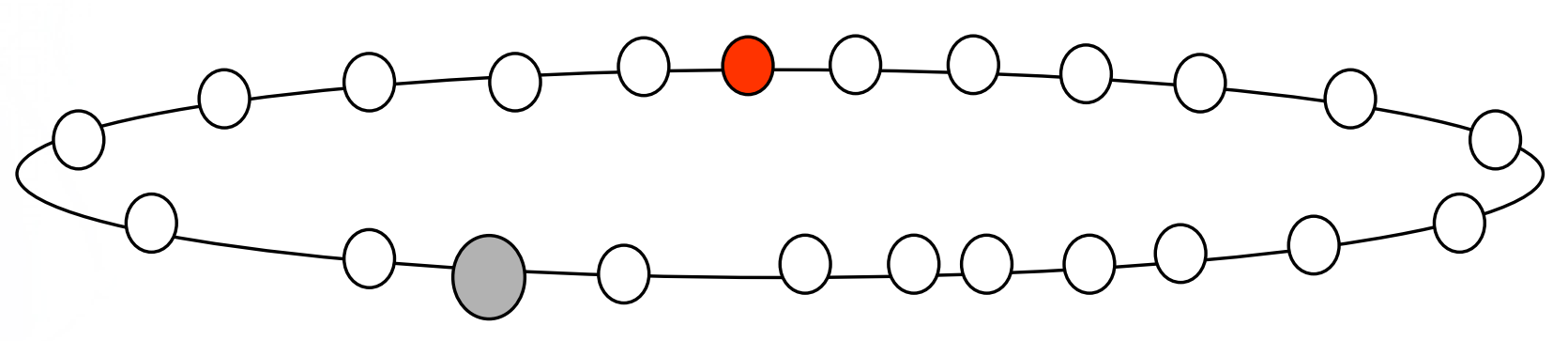

physical network topology

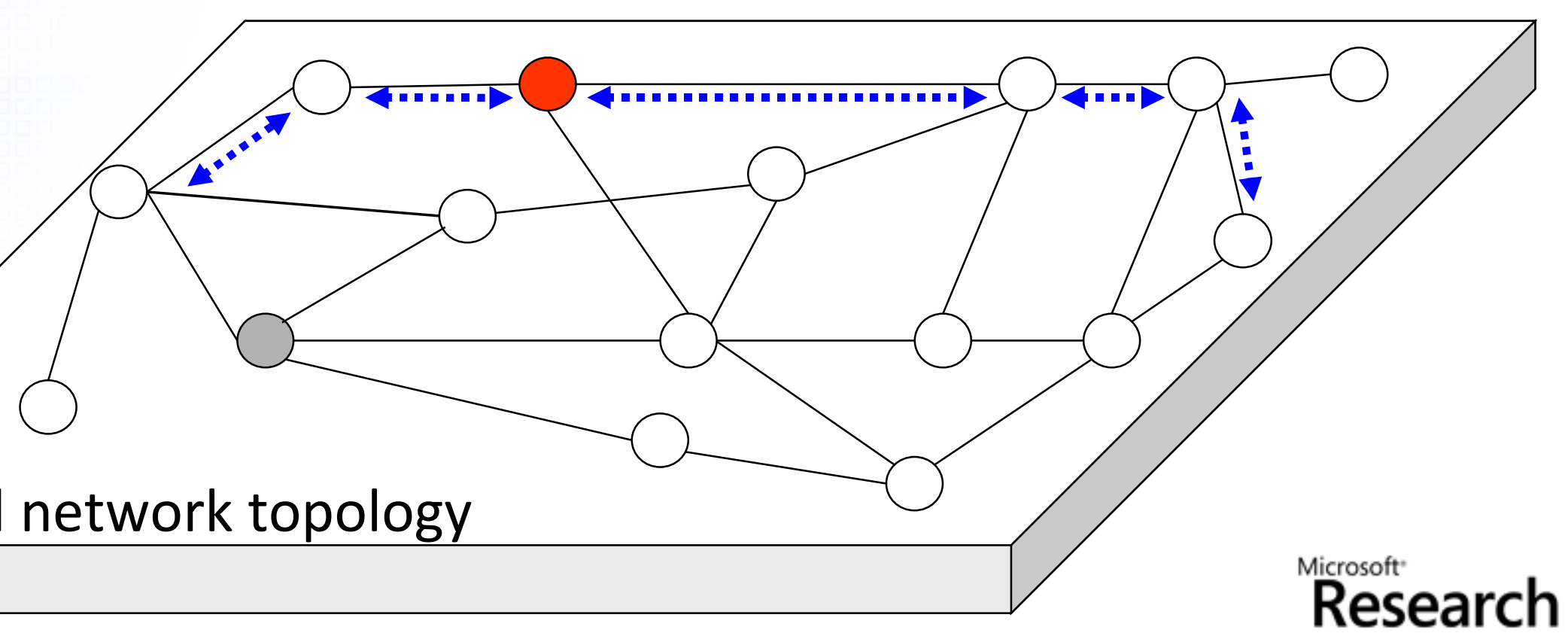




\section{VRR: Example routing}

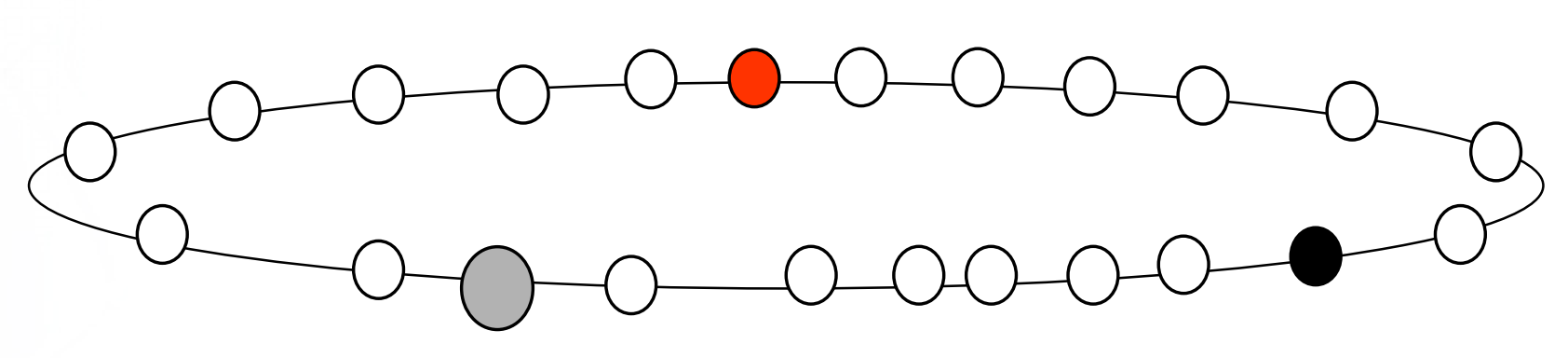

physical network topology

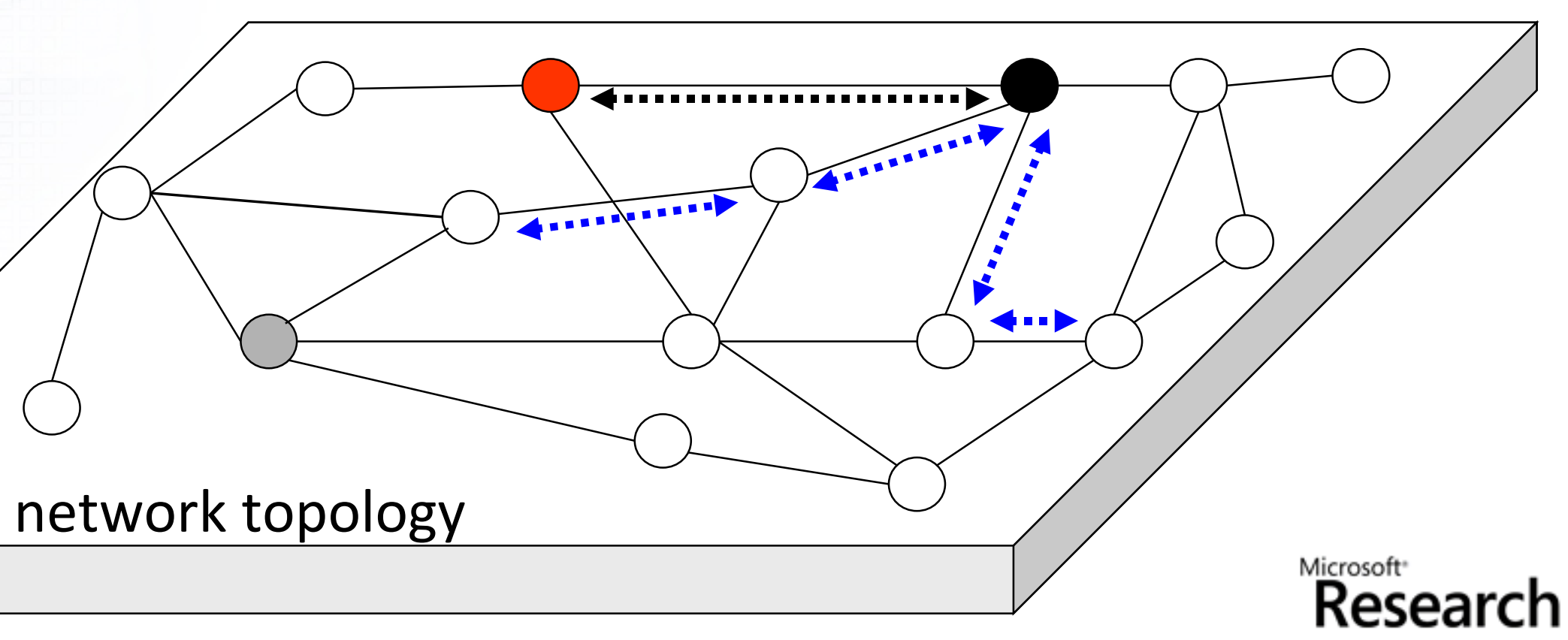




\section{VRR: Example routing}

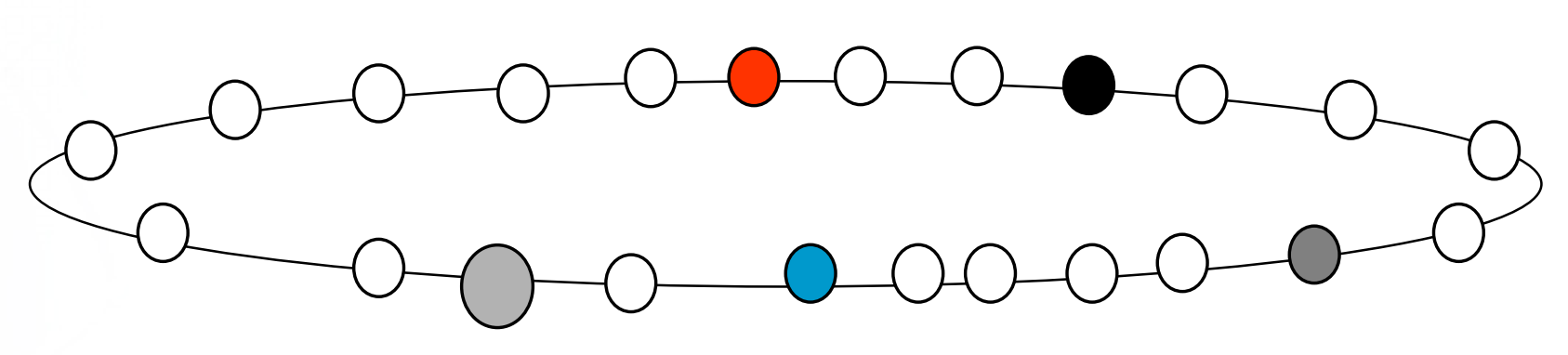

physical network topology

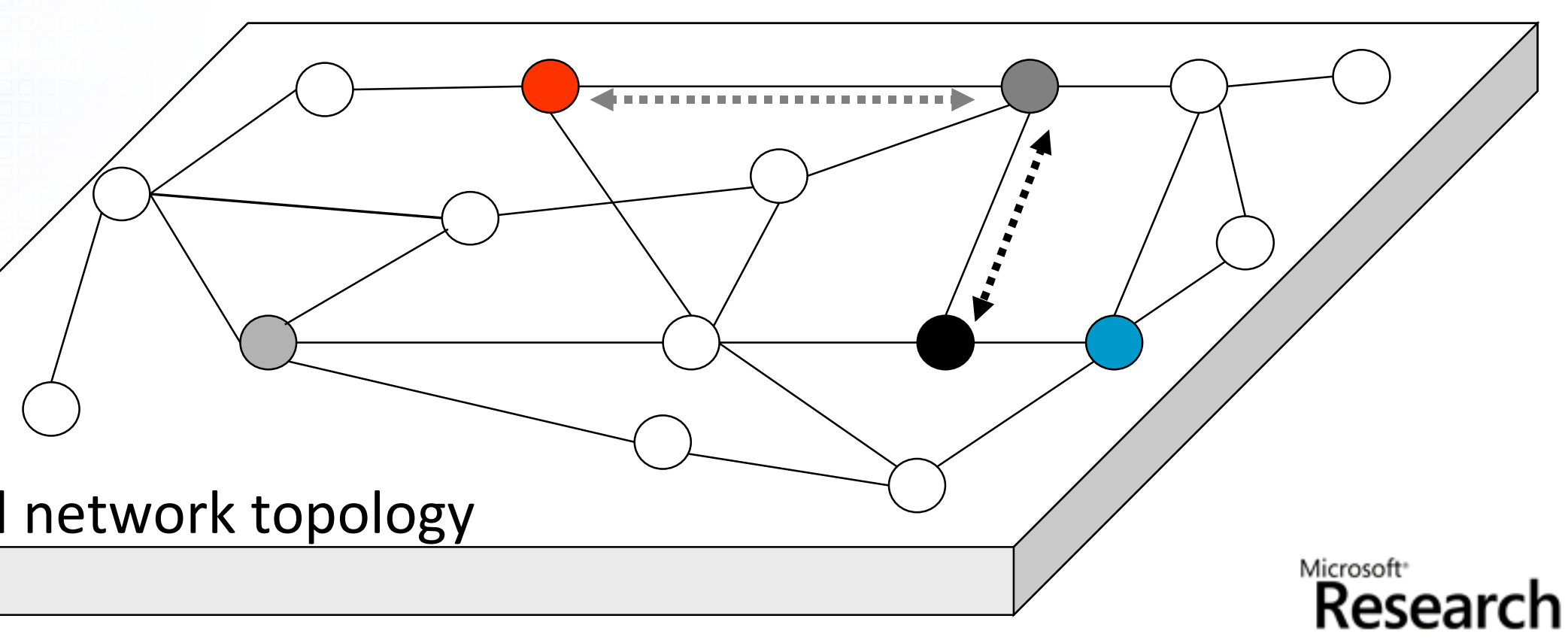




\section{VRR: Example routing}

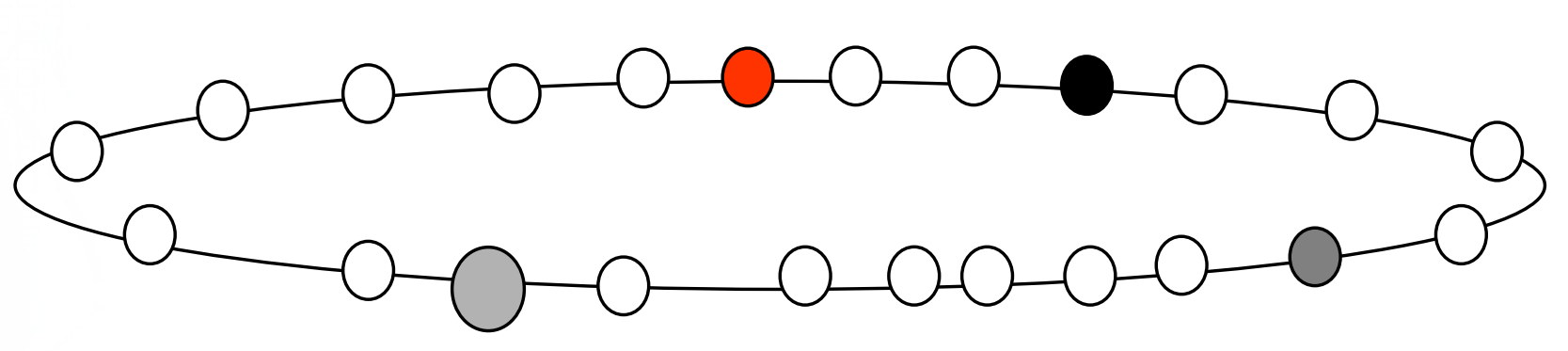

physical network topology

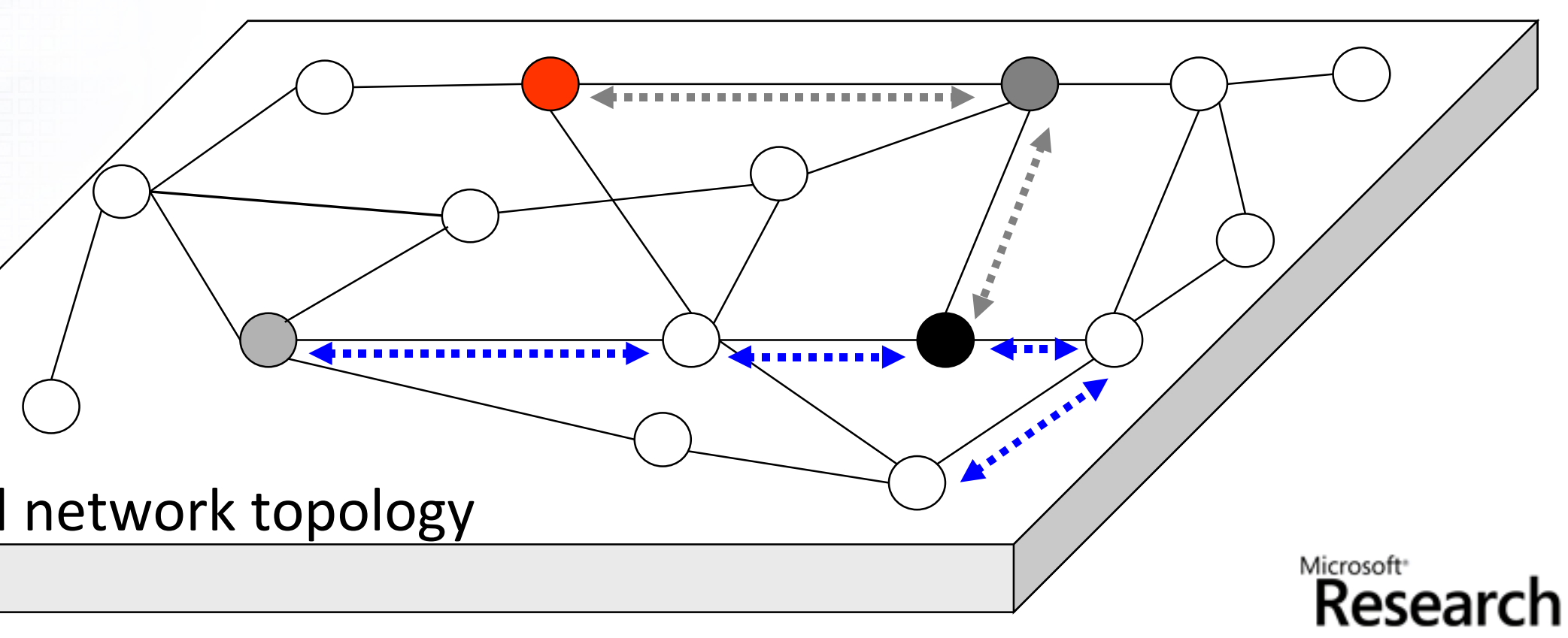




\section{VRR: Example routing}

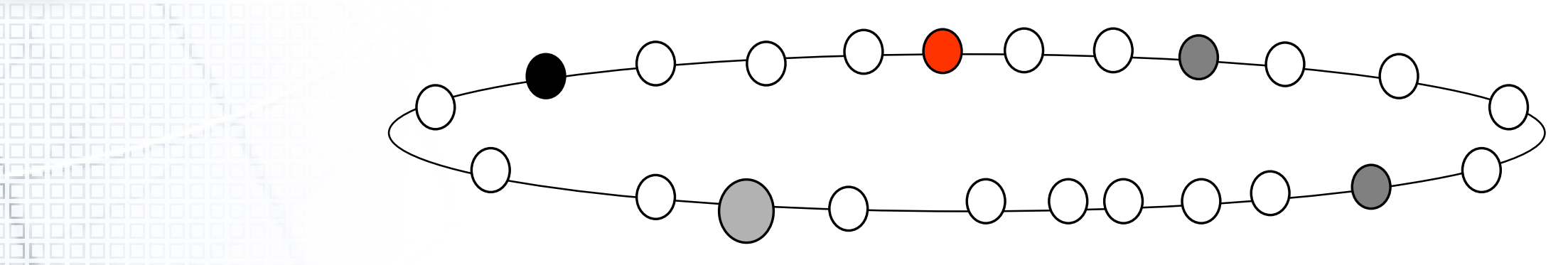

physical network topology

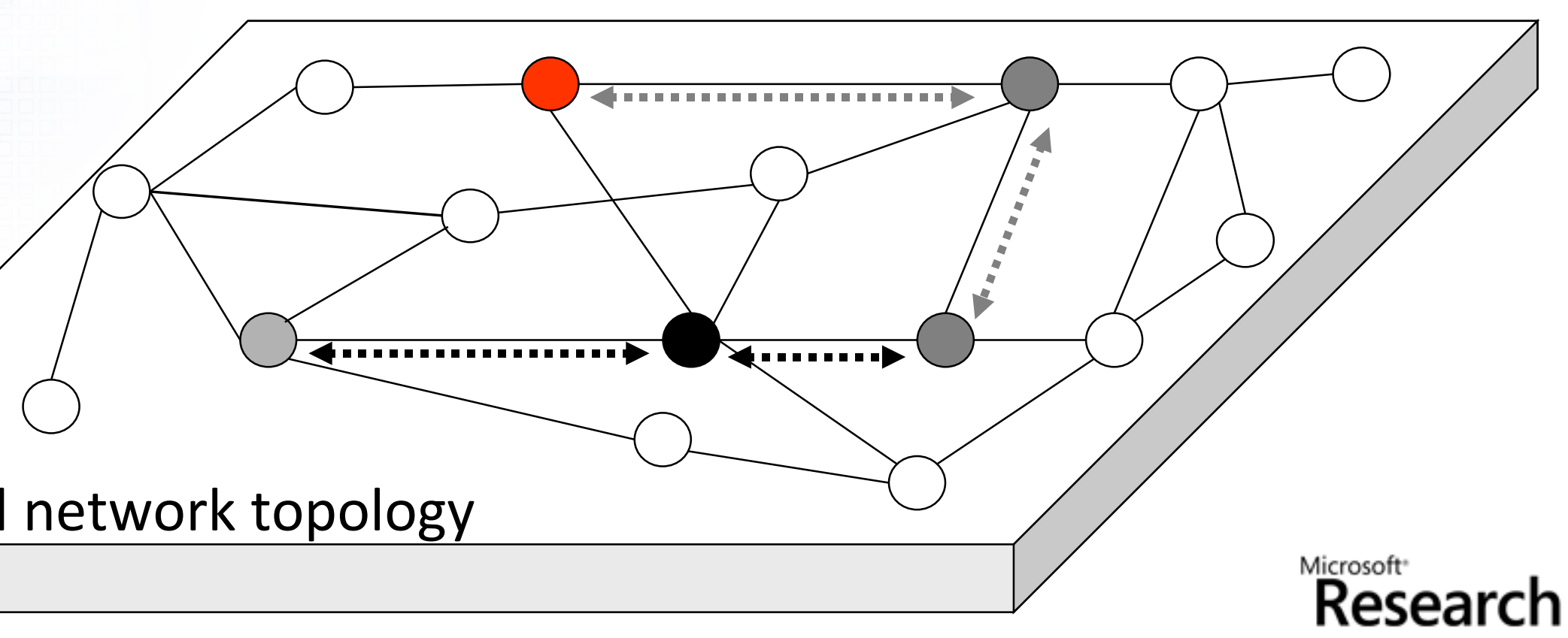




\section{VRR: Routing summary}

- Paths to virtual neighbors ensure correctness

- Stretch empirically small

- Many alternate paths to route around failures 


\section{VRR: Ring maintenance}

- No flooding

- All messages routed as described

- Single topology independent identifier

- Five message types

- hellos maintain physical neighbor set

- setups update forwarding table state along path

- setup requests ask another node to send setup

- setup replies refusal to send setup

- teardowns remove forwarding table state 


\section{VRR: Node joining}

broadcast hellos

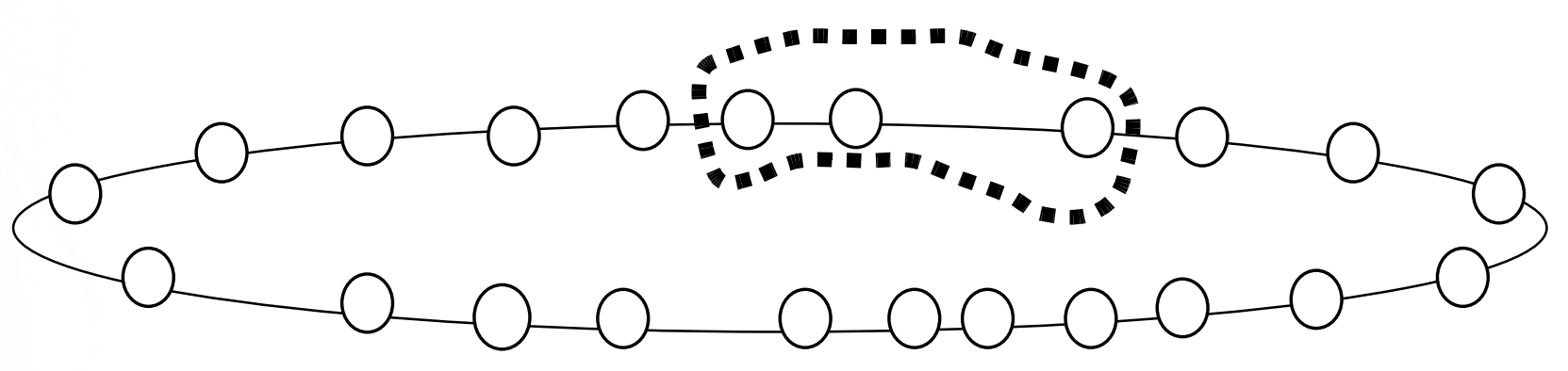

sendind phys request to $16 \mathrm{E}$

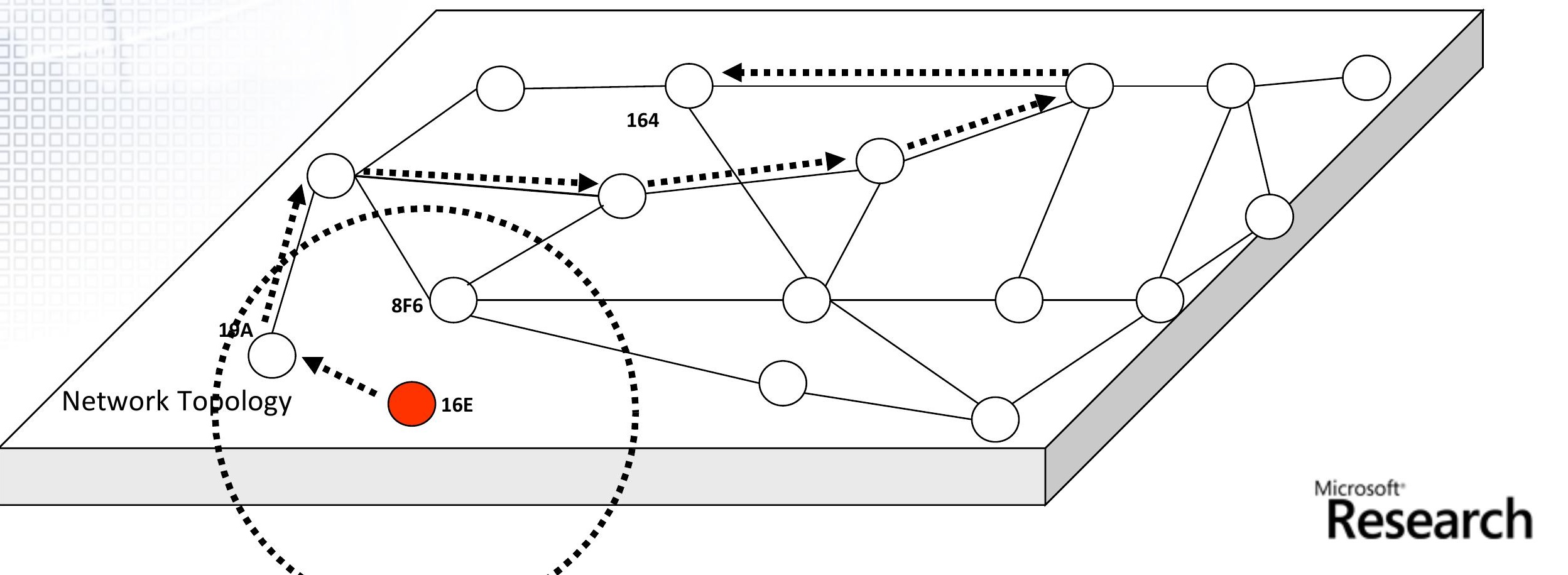




\section{VRR: Node joining}

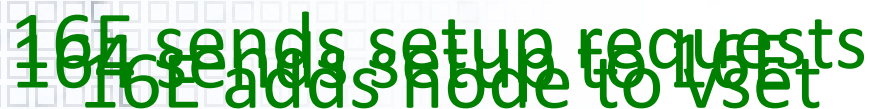

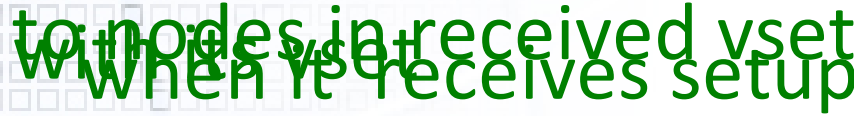

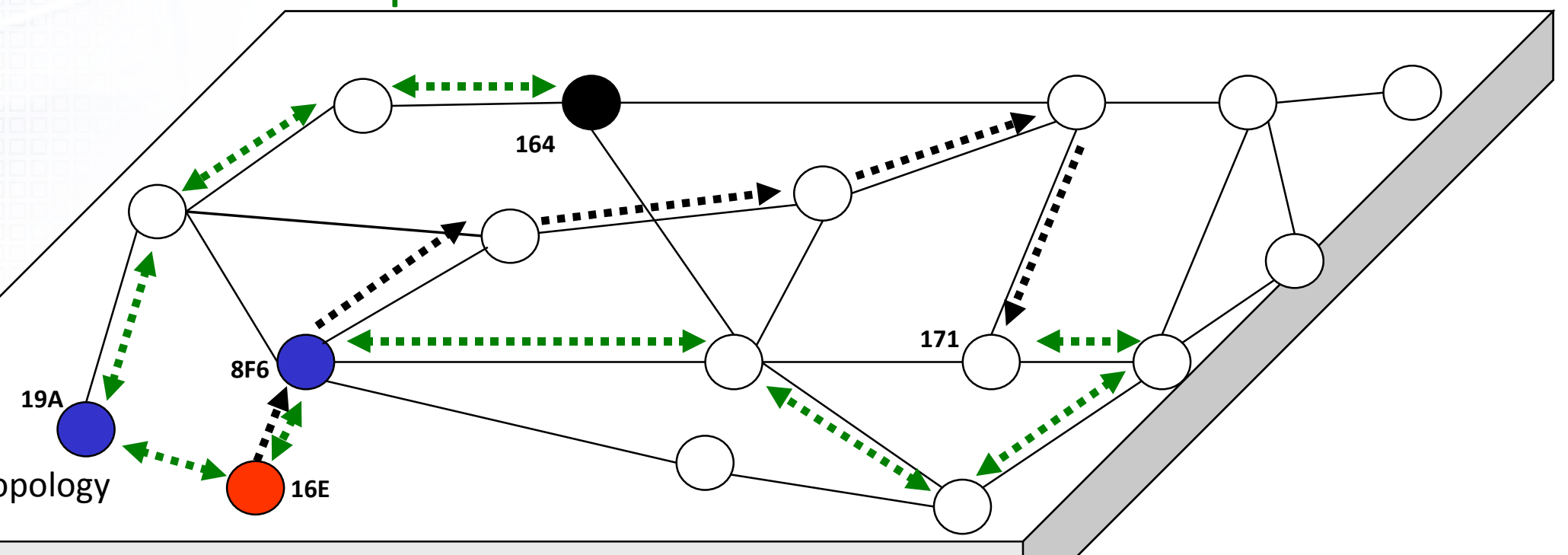

Research 


\section{VRR: Handling failures}

- Routing state is hard

- No end-to-end heartbeats

- Failures detected on missing acks or hellos

- Local repair attempted first;

- Otherwise, teardowns sent along all affected paths

- Two techniques to ensure consistency

- Symmetric failure detection and acks on teardowns

- If $x$ marks $y$ faulty, $y$ is guaranteed to mark $x$ faulty

- Lightweight optimistic transactions

- If in doubt abort (teardown) 


\section{Routing during node or link failure}

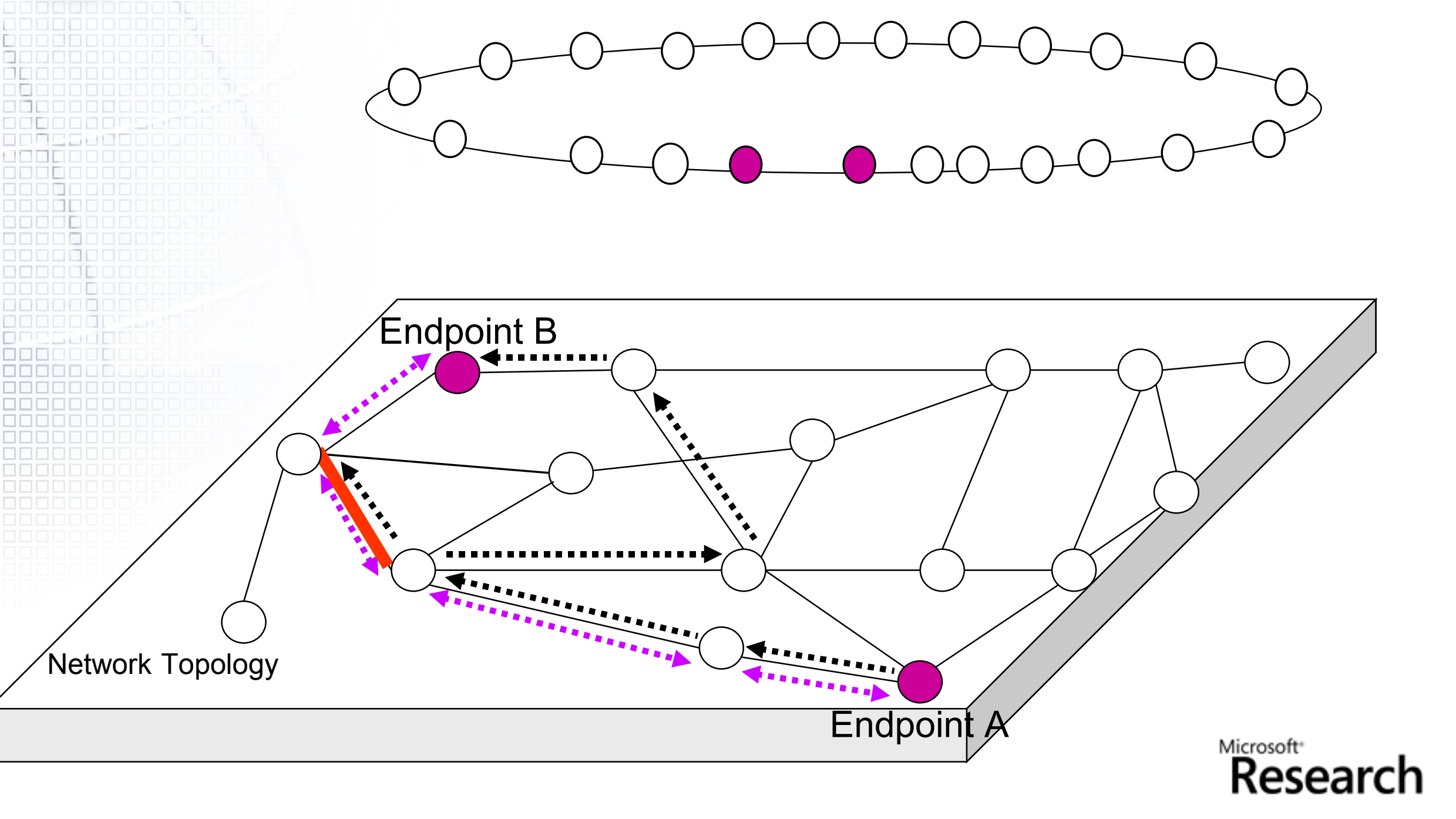




\section{A link failure example}

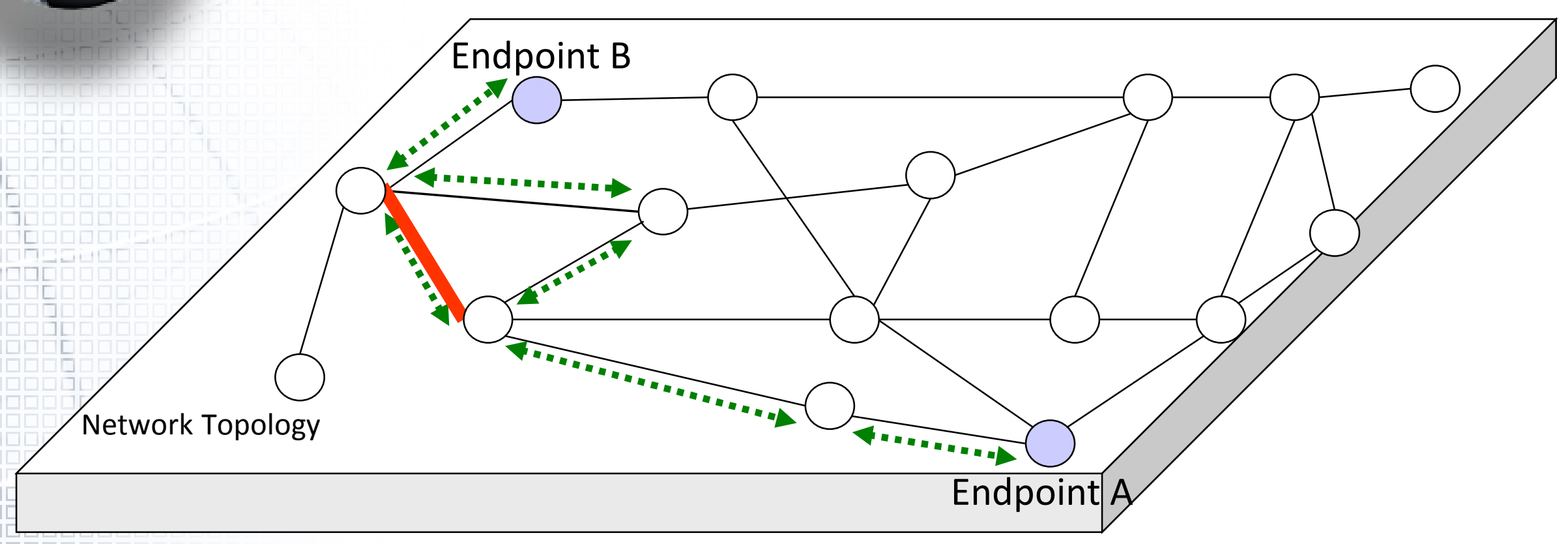

- Repair is truly local

- Only involves nodes near failed link or node

- No end-to-end path metrics

- Repair aborted if local consistency checks fail 


\section{Simulation experiments in $\mathrm{ns}-2$}

- Experiments with 802.11b MAC

- Varied network size, mobility, session lifetime

- Compared with DSDV, DSR, and AODV

- VRR performed well in all experiments

- high delivery ratios even with fast movement

- significantly lower delays with route instability 


\section{Delivery ratio: fast movement}

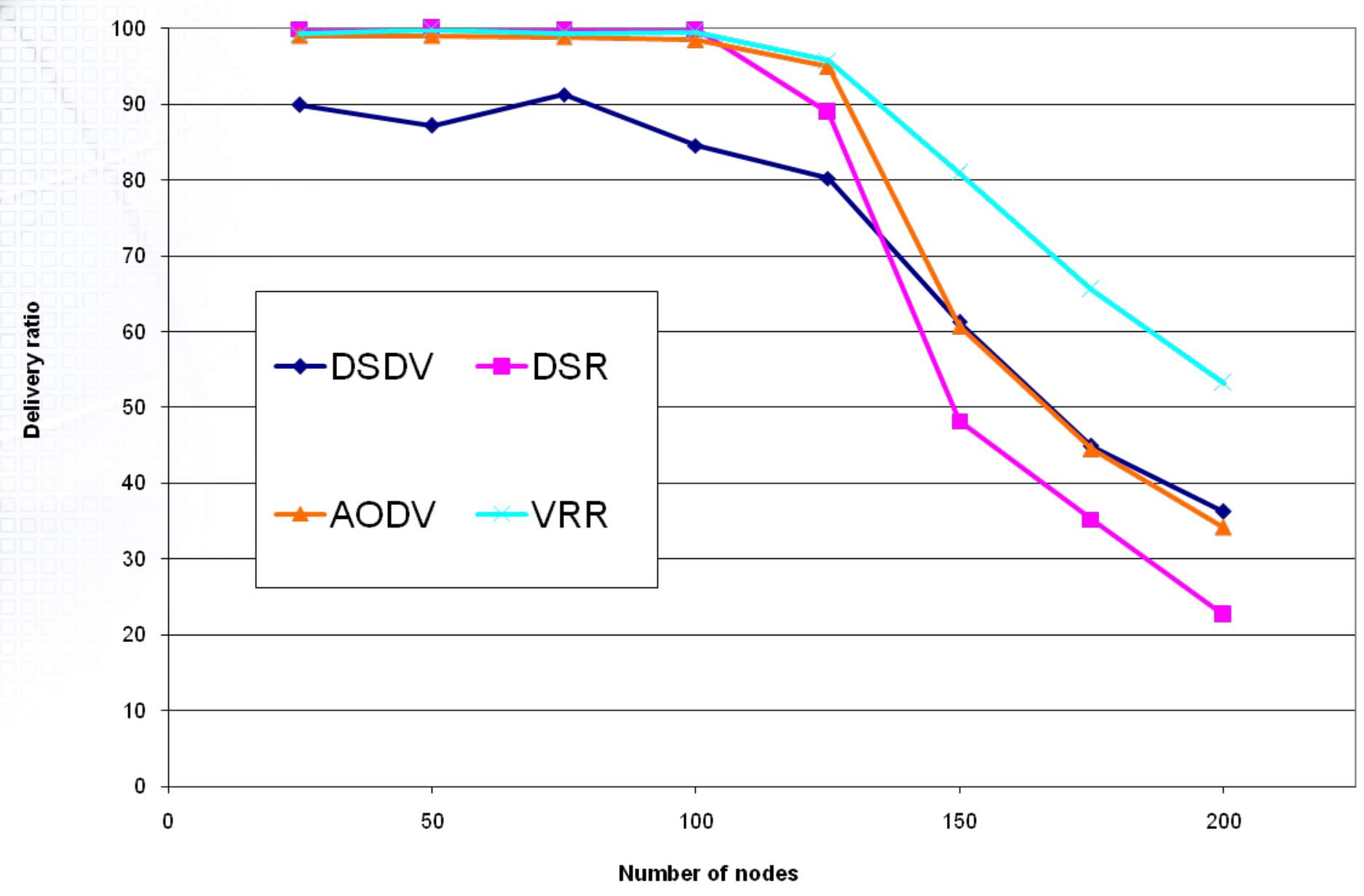

Microsoft' Research 


\section{Delay: fast movement}

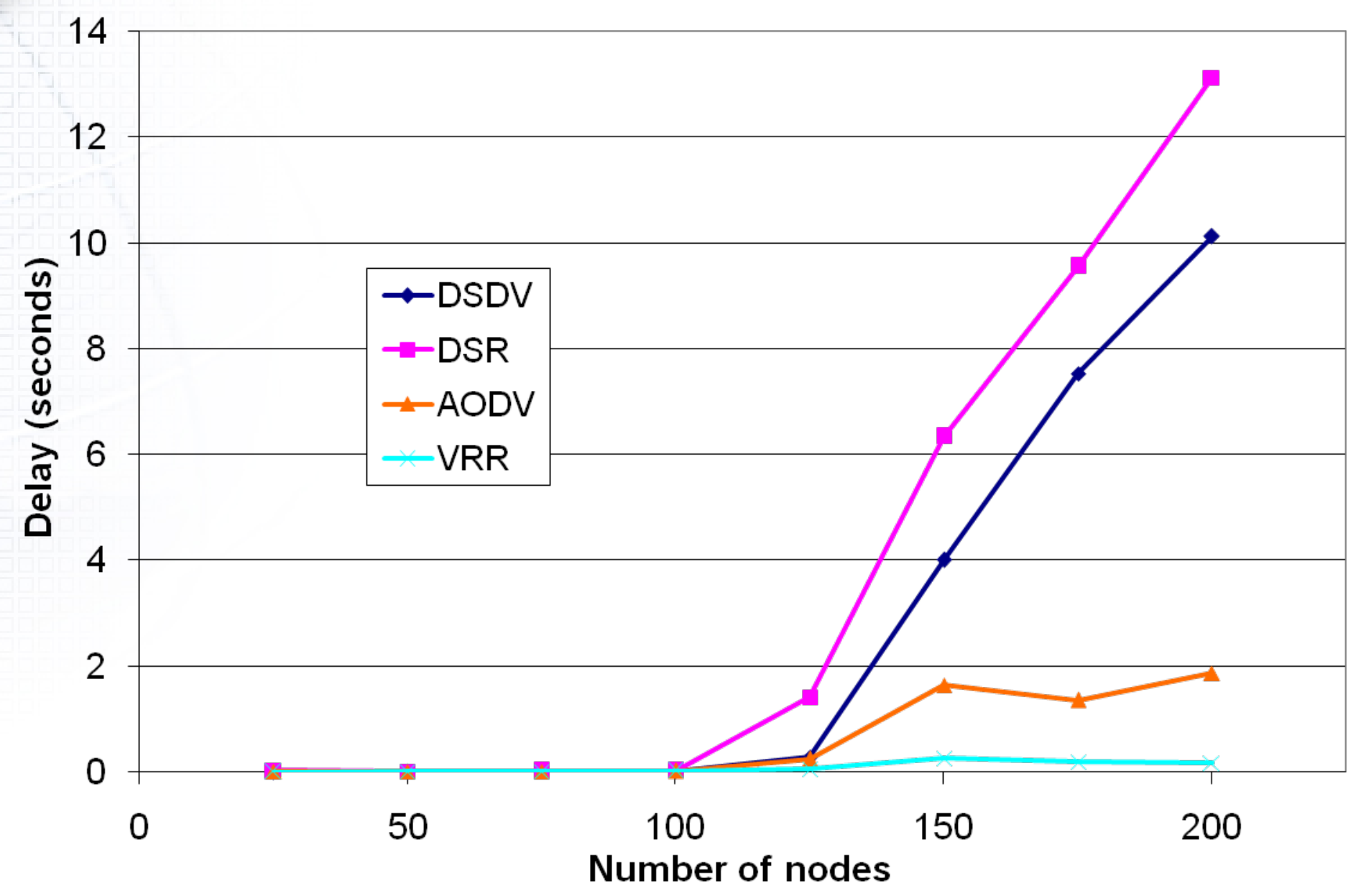




\section{Sensor network}

- Sensor network testbed

- 67 mica2dot motes in UCB building

- Comparison with BVR (coordinate-based protocol)

- Delivery ratio with mote failures 


\section{Sensor network: mote failures}

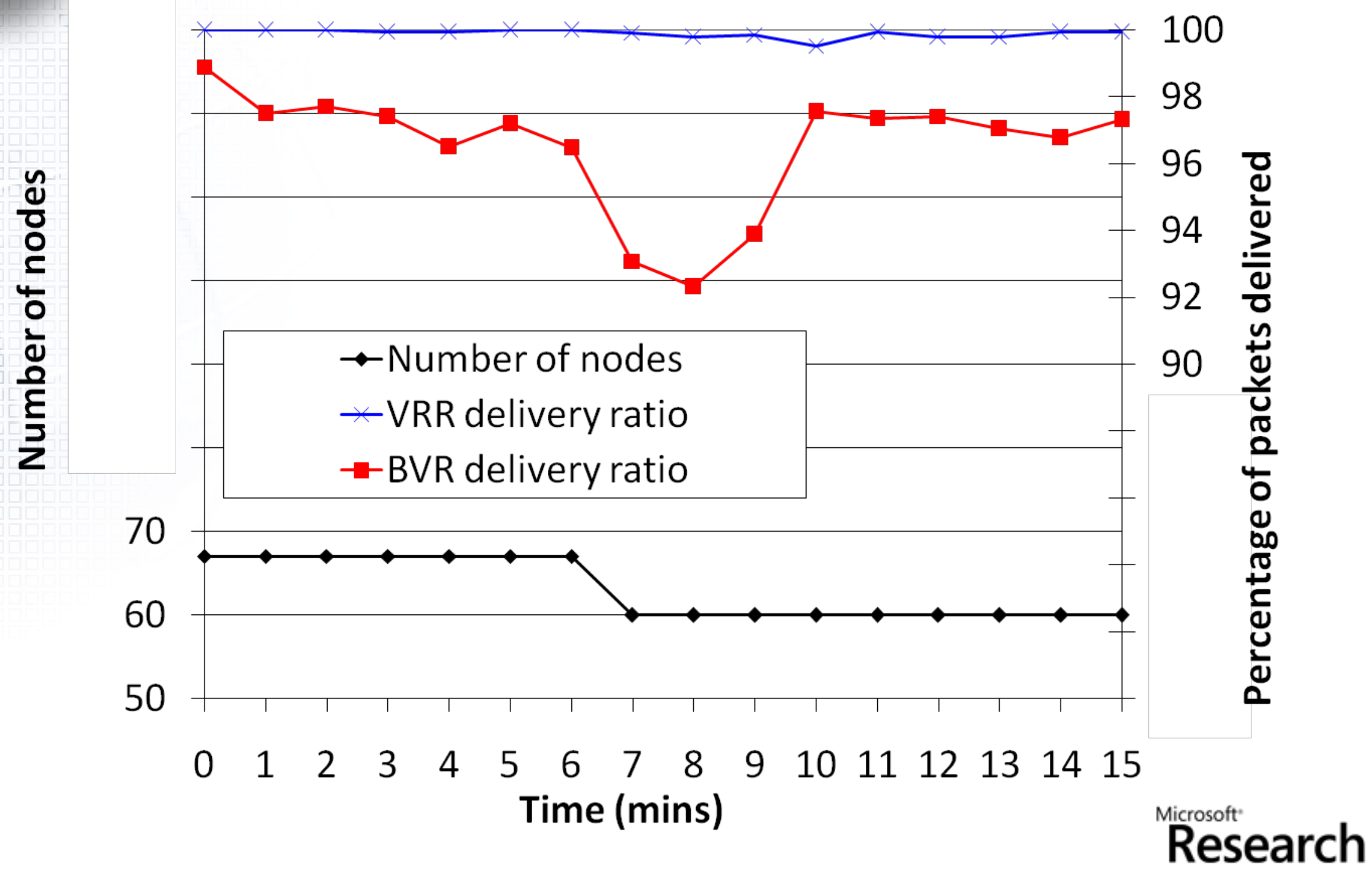




\section{Wireless office testbed}

- 30 machines running windows

- Communicate using 802.11a

- Throughput comparison with LQSR using ttcp

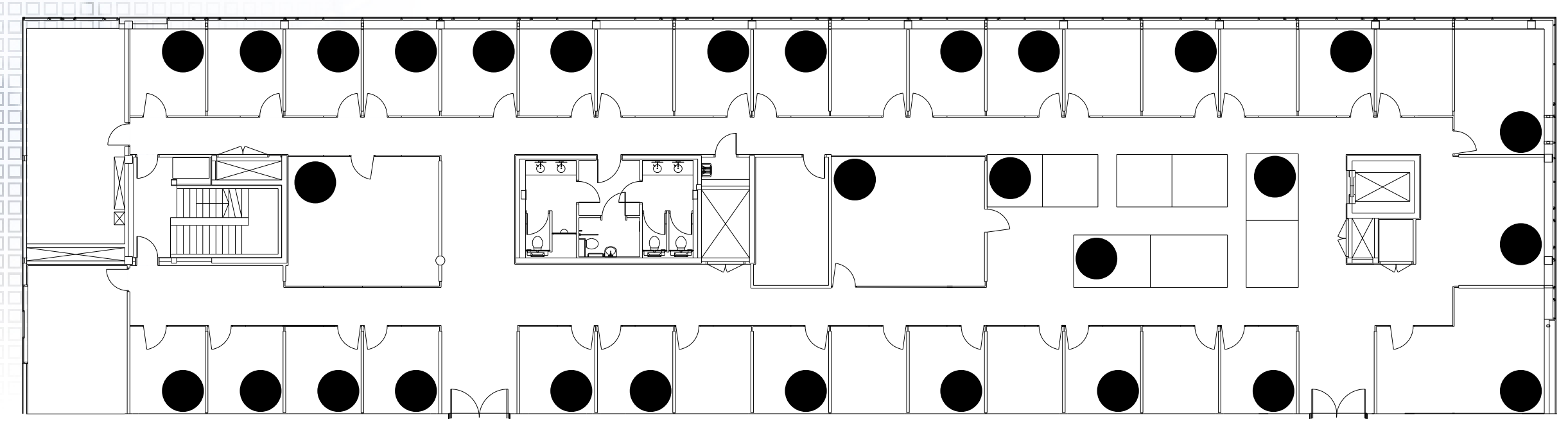




\section{Wireless office testbed: throughput}

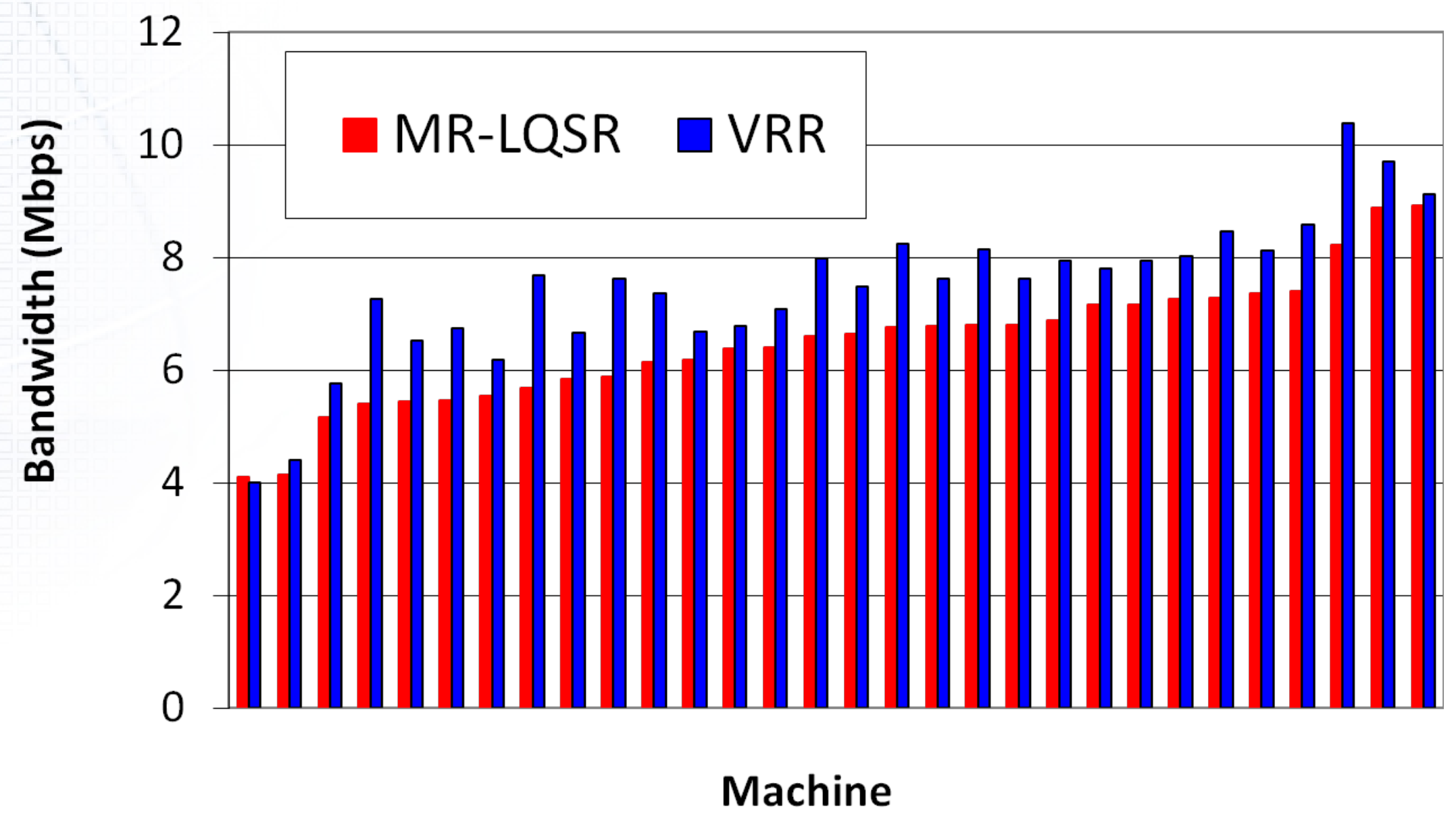

Research 


\section{Virtual Ring Routing Summary}

- Routing protocol inspired by structured overlays

- Unique (new point in the design space):

- Single identifier per node

- No flooding

- Provides DHT for free

- For more information see:

- M. Caesar, M. Castro, E. Nightingale, G. O'Shea and A. Rowstron, "Virtual Ring Routing: Network routing inspired by DHTs", Sigcomm 2006, Pisa, Italy, September 2006. 


\section{Vehicular networking}

- New challenges for scalability

- Very different characteristics

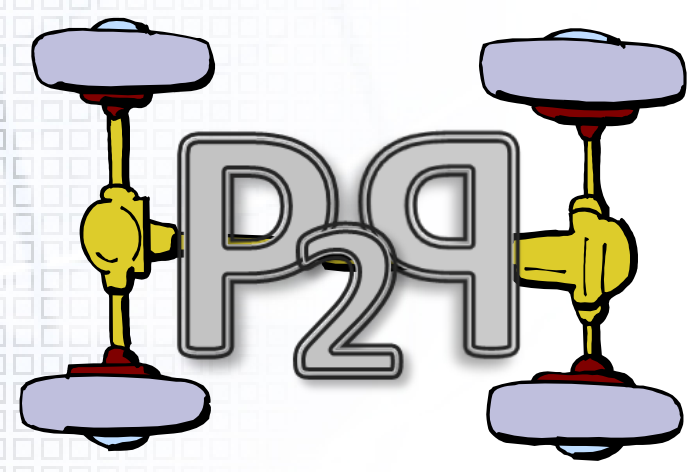




\section{Vehicle-to-Infrastructure (V2I)}
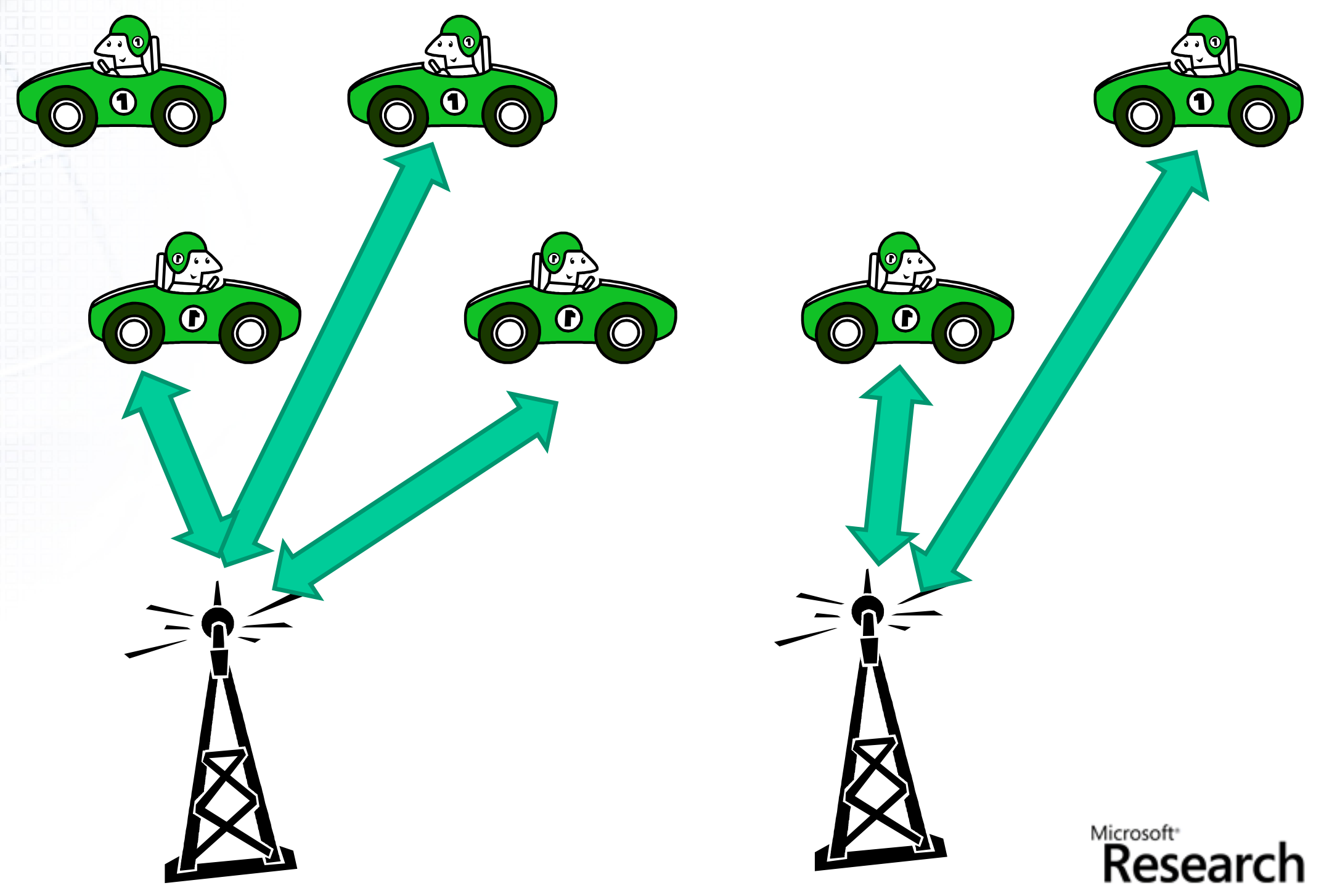


\section{Vehicle-to-Vehicle (V2V)}

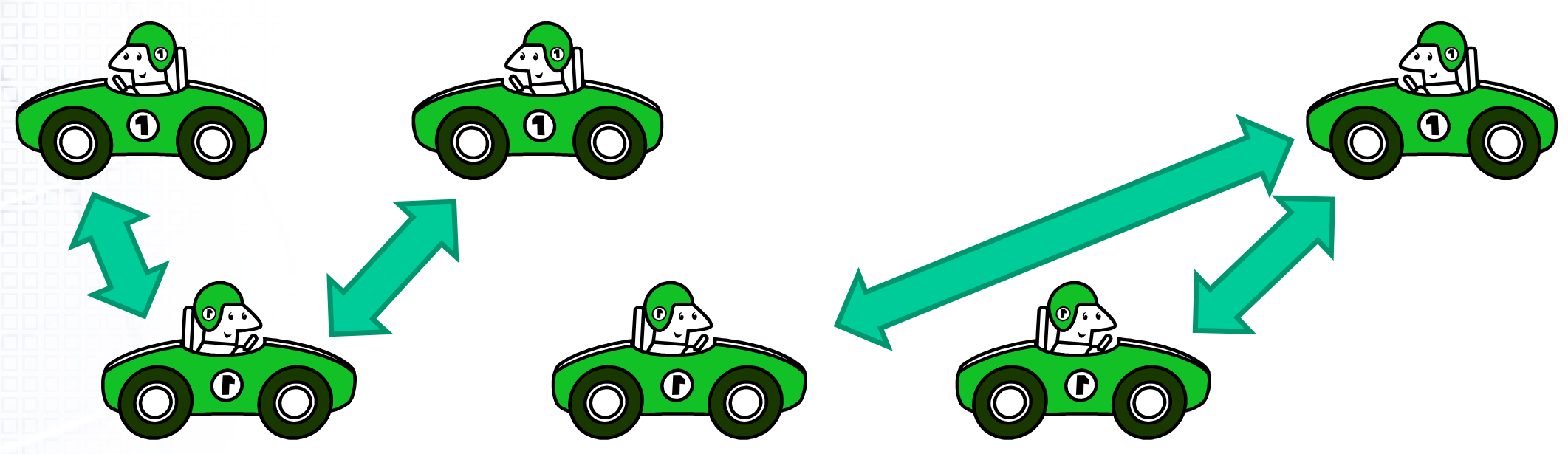

- Car manufacturers interested and "driving":

- 802.11p (Dedicated short range communications)

- Intelligent Transport Systems

- "What is the car in front doing?"

- But then..... 


\section{The disruptive device......}

- SatNav (TomTom)

- Dual connectivity model:

- Bluetooth to mobile phone

- USB-to-computer (new map > 1Gb)

- Download data for premium services (Home service)

- TomTom Map Share (Web 2.0 app)

- TomTom QuickFix (Assisted GPS)

- TomTom Updates

- "Connectivity key"

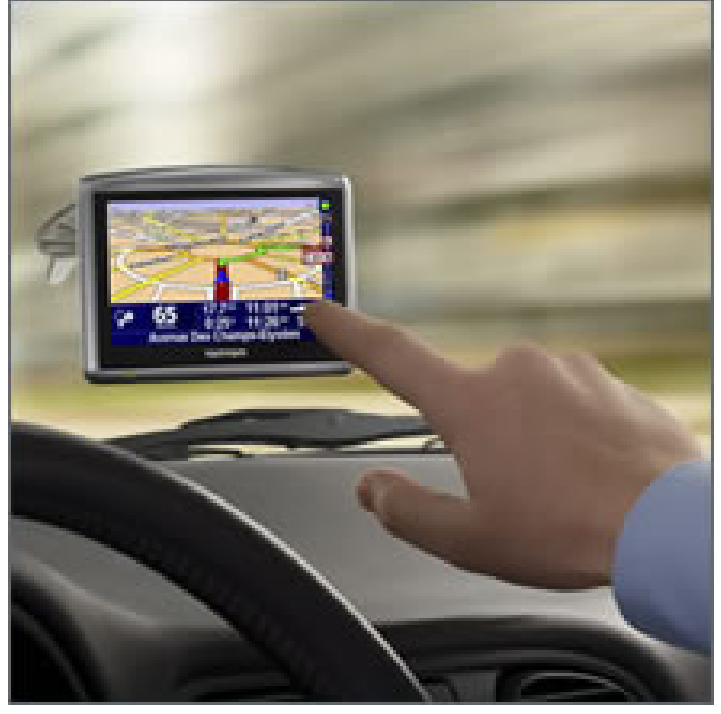

Microsoft: Research 


\section{Personal Navigation Devices}

"Hand held" or In-dash!

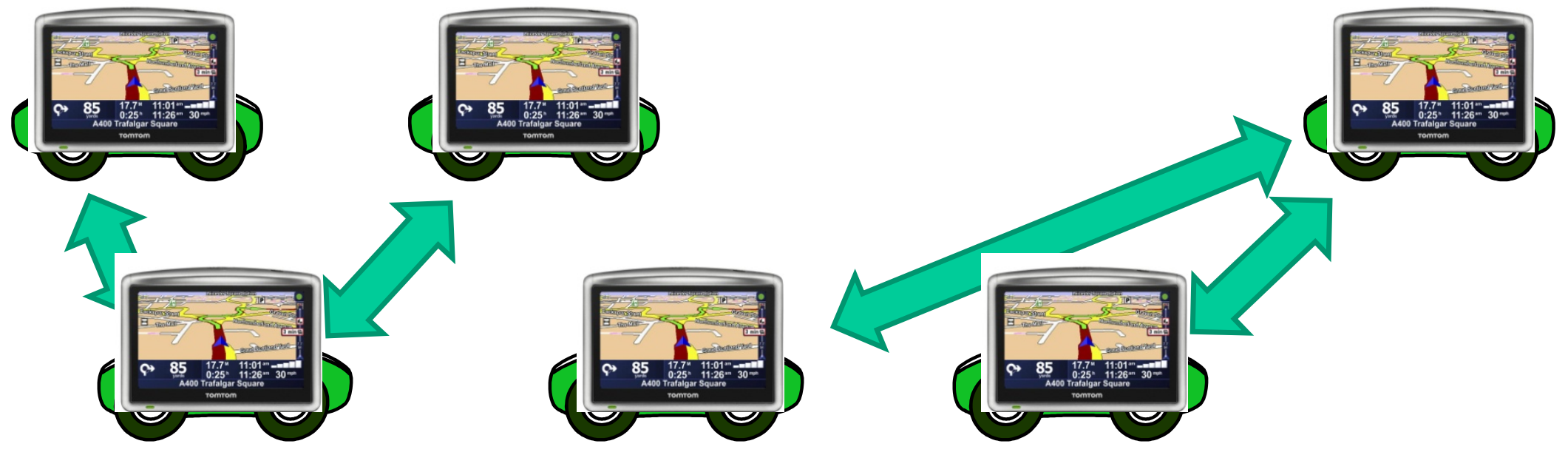




\section{PVRP: Practical Vehicular Routing Protocol Joint work with G. Pau and P. Lutterotti (UCLA)}

- Multi-hop vehicle-to-infrastructure

- Route to fixed access points

- Multi-hop vehicle-to-vehicle

- Route to specific vehicle or a service

- Why do we need another routing protocol? 


\section{Greedy Routing}

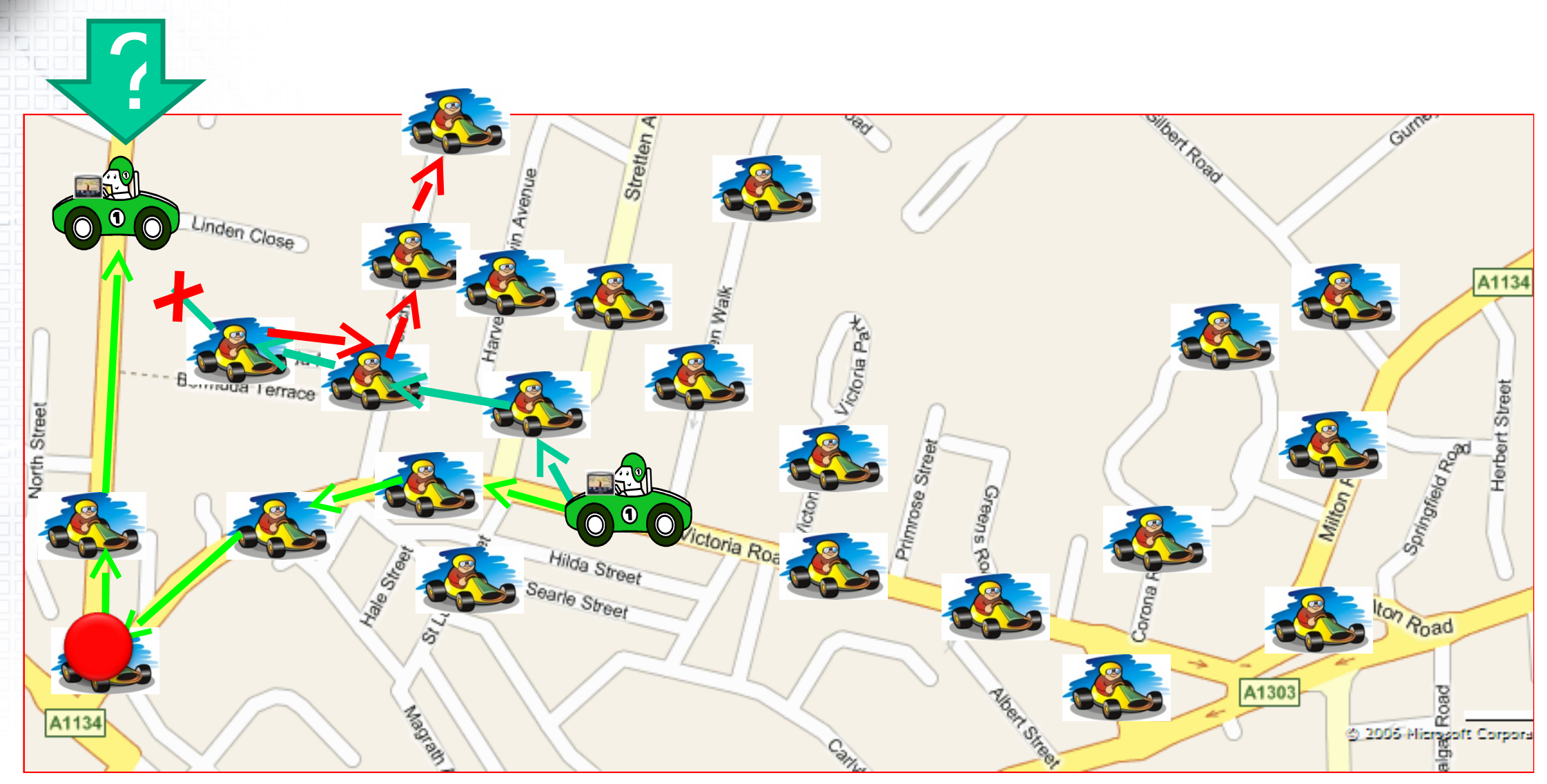

"Research 


\section{Biscovery: Sparse}

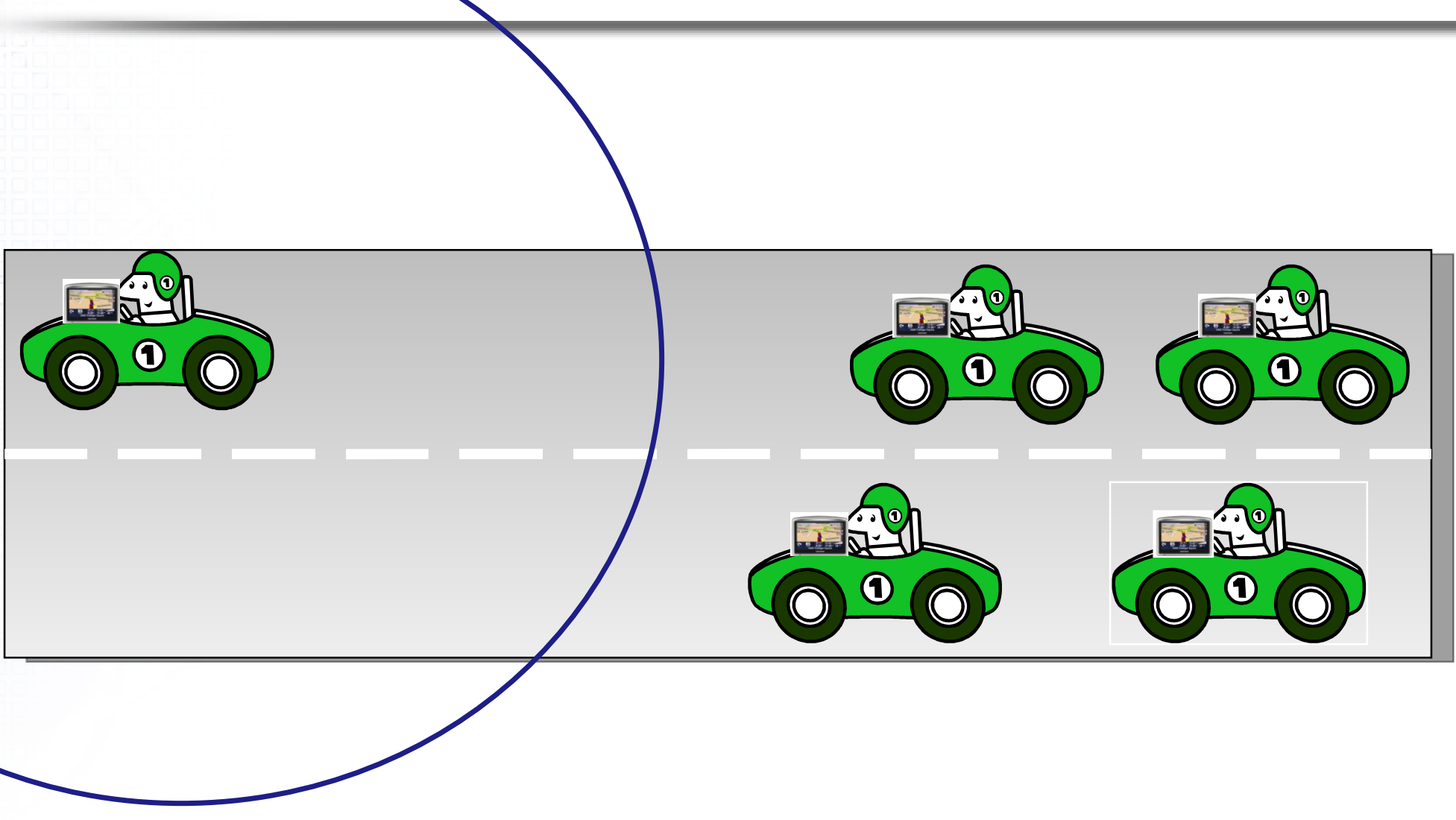

Sparse more common than dense $\rightarrow$ "delay" tolerant protocol 


\section{The PVRP approach}

- Assume nodes have:

- Digital maps (e.g. NavTech digital maps)

- 802.11a/b/g WiFi (or equivalent)

- GPS system

- Perform routing and discovery in map space not physical topology

- Opposite to VRR 


\section{Assume nodes maintain one-hop topology information}
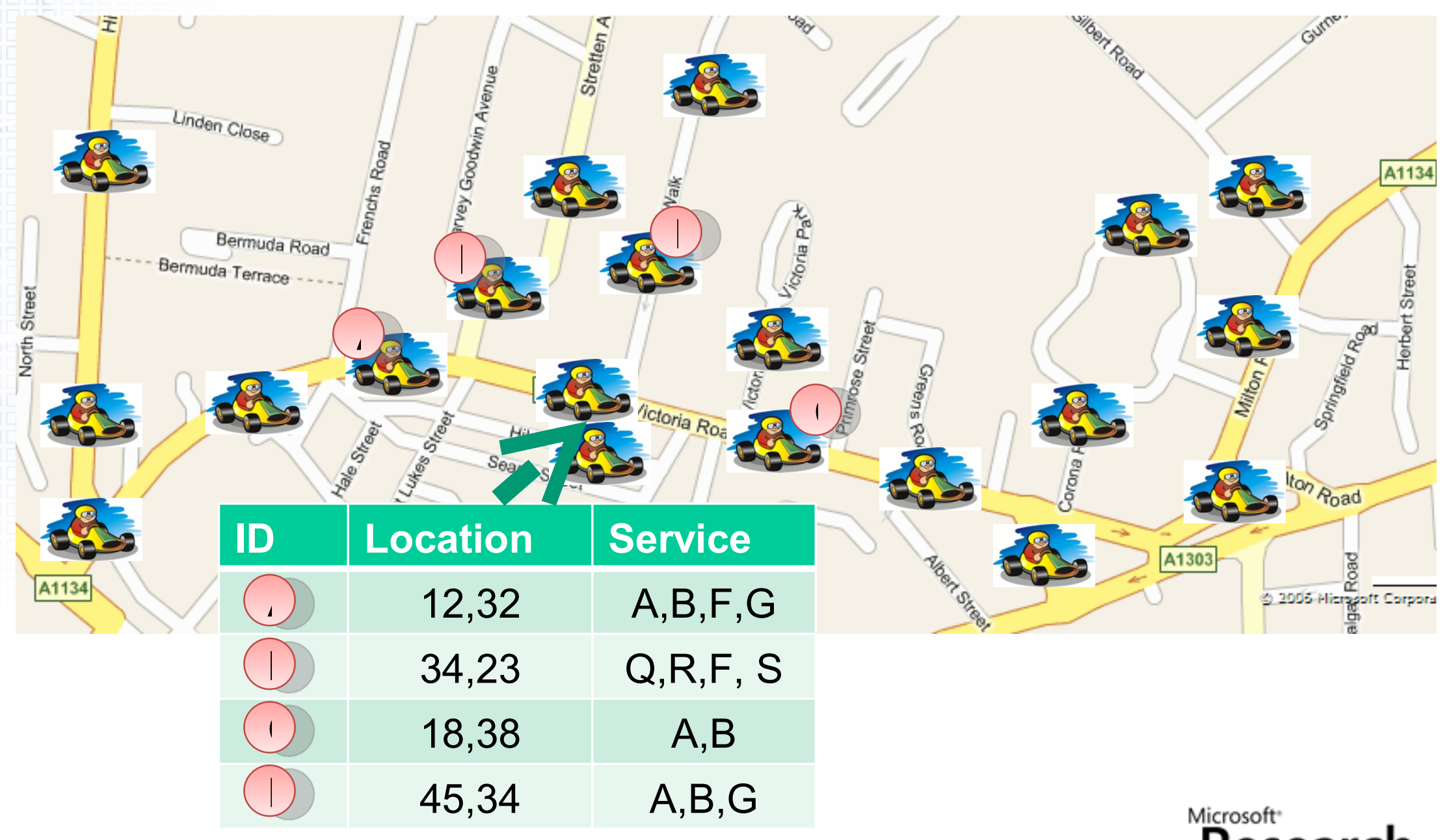

Research 


\section{Discovery: Exploit map}

- Avoid pure flood in physical topology

- Exploit map to ensure good exploration

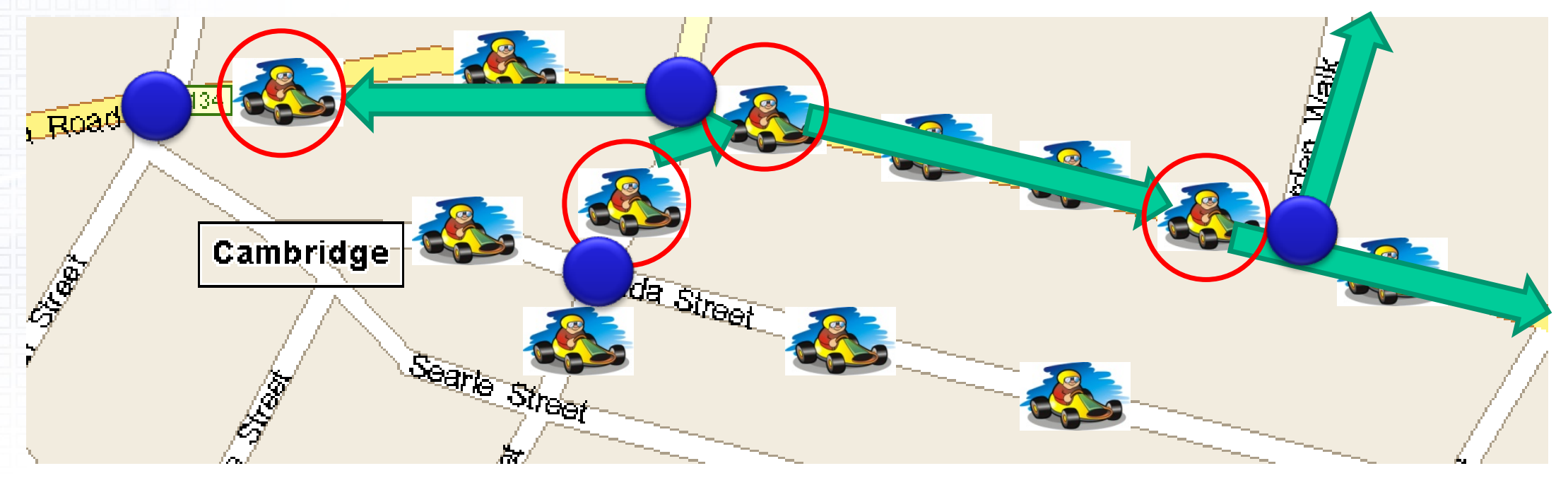




\section{Map-based source routing}

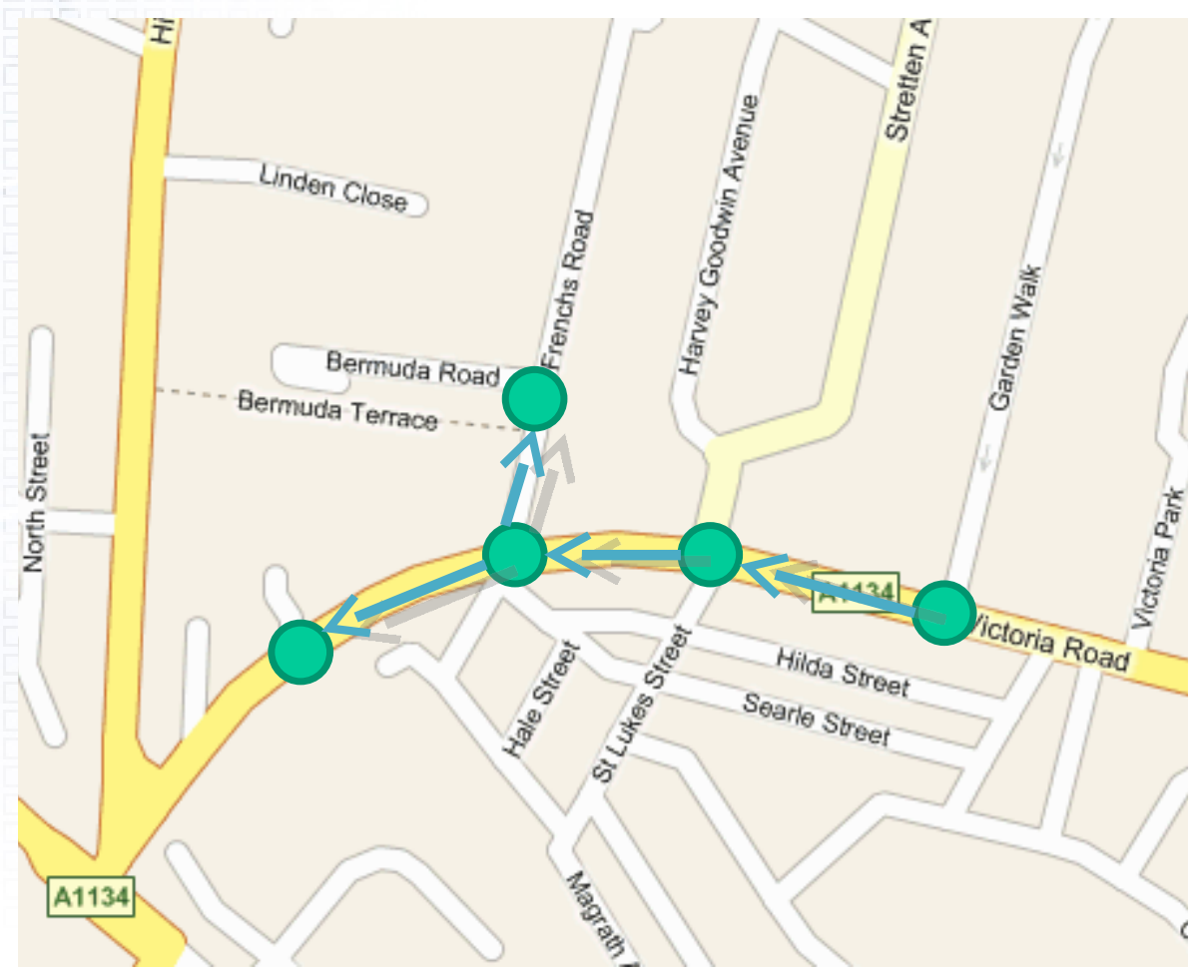

Packet: $<$ Src $><$ Dest $><$ etc $>$ $<$ Path:

Victoria Road -> Garden Walk Go W Victoria Road -> Harvey Avenue Go W Victoria Road -> Frenchs Road Go SW $>$

Packet: $<$ Src $><$ Dest $><$ etc $>$ $<$ Path:

Victoria Road -> Garden Walk Go W Victoria Road -> Harvey Avenue Go W Victoria Road -> Frenchs Road Go N $>$

Note: Between any two Junctions the packet may traverse several network hops 


\section{Preliminary results}

- Currently understanding the design choices and their impact:

- Path selection metrics:

- Average density,

- Max lowest density, etc

- Junction selection metrics:

- Select the important junctions

- Comparing against delay tolerant greedy algorithm 


\section{Preliminary results}

- Currently evaluating PVRP using:

- "Simple" simulator (versus Flood)

- QualNet simulator (versus AODV/DSR/GPSR)

- Stand alone implementation (keeping us honest!)

- Using mobility traces for Portland

- Results today generated using simple simulator

- Comparing PVRP with "Flood"

- Flood represents best that AODV/DSR/GPSR could do

- Results using "realistitc" mobility trace

- 59 runs randomly selected static end-points with distance between $250 \mathrm{~m}$ and $750 \mathrm{~m}$

- Across all runs only 27 experiments delivered packets 


\section{Delivery ratio: Preliminary results}

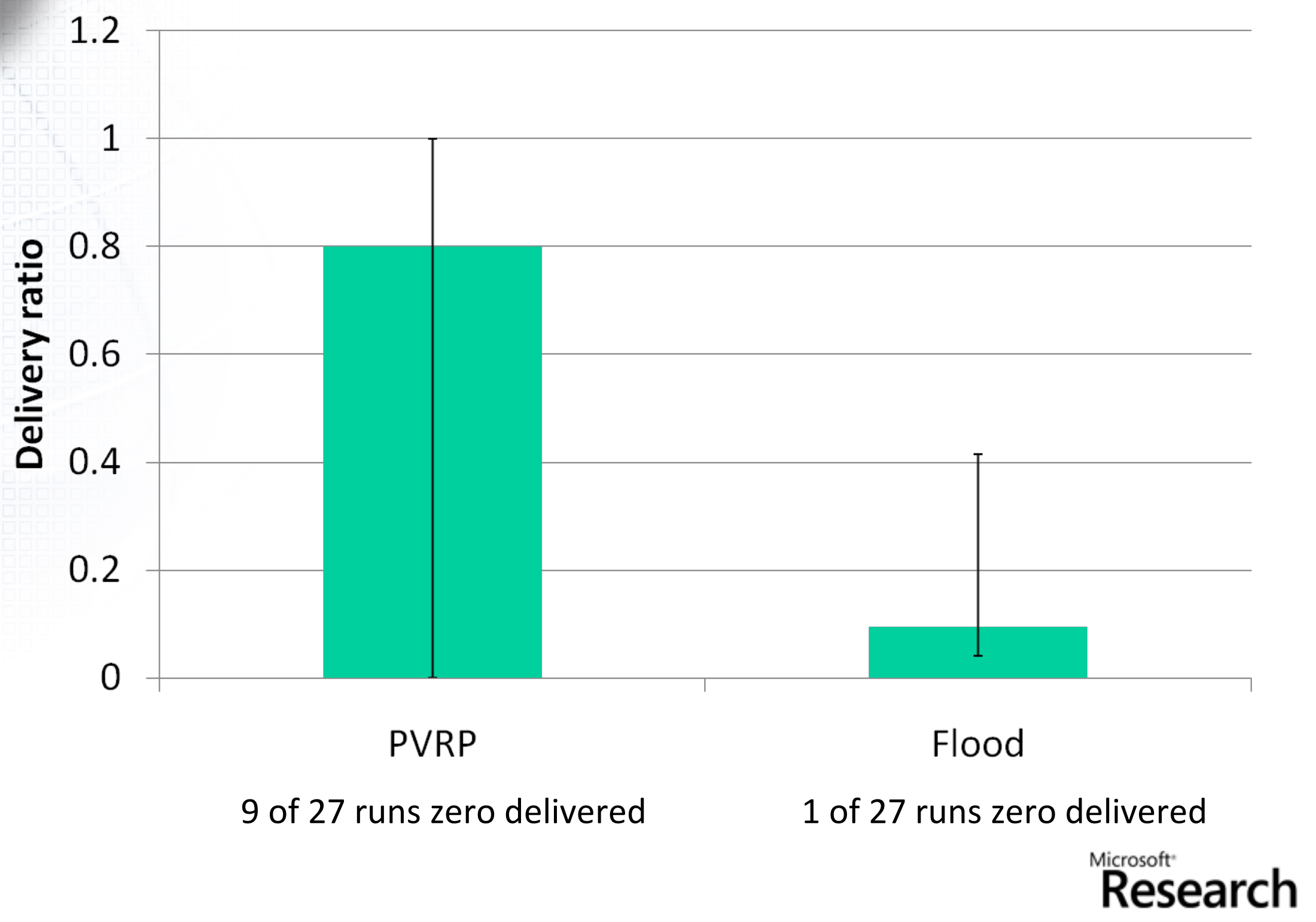




\section{Delay: Preliminary results}

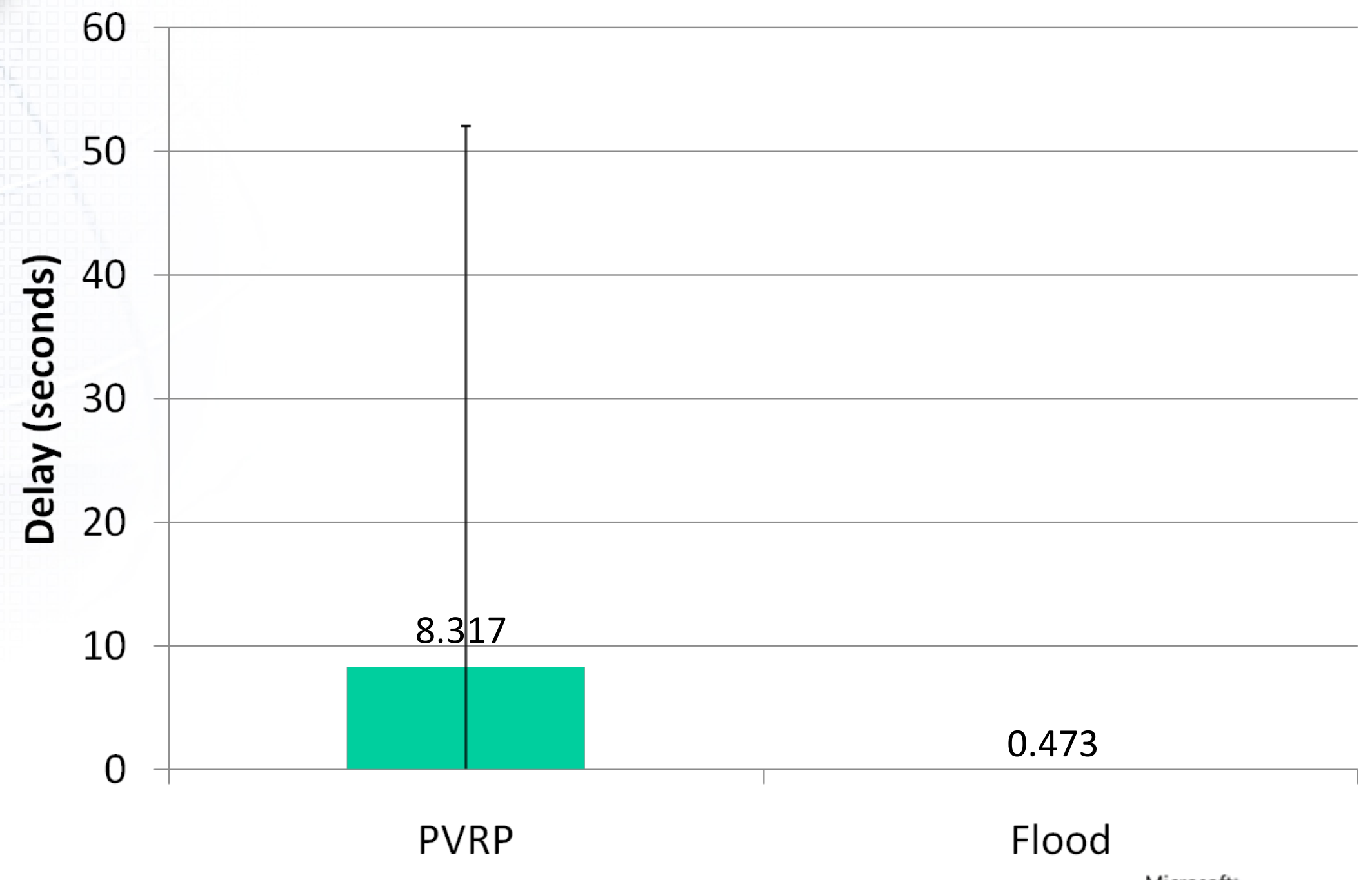




\section{PVRP Summary}

- Practical multi-hop routing protocol for vehicular networks

- Exploits digital maps rather than just the physical network topology

- To work over partitioned networks 


\section{Thank you}

http://research.microsoft.com/ antr 